\title{
Spacelike Localization of Long-Range Fields in a Model of Asymptotic Electrodynamics
}

\author{
Andrzej Herdegen and Katarzyna Rejzner
}

\begin{abstract}
A previously proposed algebra of asymptotic fields in quantum electrodynamics is formulated as a net of algebras localized in regions which in general have unbounded spacelike extension. Electromagnetic fields may be localized in 'symmetrical spacelike cones', but there are strong indications this is not possible in the present model for charged fields, which have tails extending in all space directions. Nevertheless, products of appropriately 'dressed' fermion fields (with compensating charges) yield bi-localized observables.
\end{abstract}

\section{Introduction}

In this paper we continue the investigation of the infrared structure of quantum electrodynamics based on an algebraic model proposed earlier by one of us (see [1] and papers cited therein; see also [2]). This model is supposed to describe asymptotic fields in the quantum Maxwell-Dirac system, including the Gauss' law constraint (as opposed to the crossed product of free fields).

In a recent paper [3], this model was investigated in respect of the localization properties of fields. It was shown that one needs an extension of the localization regions: infrared/charge structure is encoded in unbounded regions. It was argued that from the point of view of scattering theory, the natural choice for extended localization regions consists of 'fattened lightcones', unions of intersecting: a future- and a past-lightcone. The test functions of electromagnetic fields have well-defined asymptotes encoding the information on the long distance structure.

In the present article we show that the algebra can be localized in any 'time-slice' which is fattening under constant inclination towards infinity. In addition, the localization of electromagnetic field may be restricted to 'fattened symmetrical spacelike cones': the unions of a spacelike cone and its reflection with respect to a point in its inside. Similar restriction seems to be ruled out, 
even asymptotically, for charged fields. This seems to contradict general wisdom on the expected behavior of fields in full electrodynamics, see e.g. the assumptions on which Buchholz [5] bases his selection criterion of representations in quantum electrodynamics. Whether this points to some incompleteness of the model is an open question; see the discussion at the beginning of Sect. 5 below and in Sect. 6. On the other hand, we show that in the present model, in agreement with the general expectation, one can superpose two appropriately "dressed" Dirac fields carrying opposite charges to obtain a local observable.

This article should be regarded as a continuation of references [1] and [3], and we refer the reader to these references for more detail and a wider background. However, we briefly summarize notation and the formulation of the model in the next two sections. We obtain spacelike localization of fields in Sects. 4 and 5, and discuss the results in concluding Sect. 6.

\section{Geometrical Preliminaries}

The geometry of the spacetime is given by the affine Minkowski space $\mathcal{M}$. If a reference point $O$ is chosen, then each point $P$ in $\mathcal{M}$ is represented by a vector $x$ in the associated Minkowski vector space $M$ according to $P=O+x$. We mostly keep $O$ fixed and use this representation. The Minkowski product is denoted by a dot, $x \cdot y$, and we write $x^{2}=x \cdot x$. If a Minkowski basis $\left(e_{0}, \ldots, e_{3}\right)$ in $M$ is chosen, then we denote $x=x^{a} e_{a}$. We also then use the standard multiindex notation $x^{\alpha}=\left(x^{0}\right)^{\alpha_{0}} \ldots\left(x^{3}\right)^{\alpha_{3}},|\alpha|=\alpha_{0}+\cdots+\alpha_{3}, D^{\beta}=\partial_{0}^{\beta_{0}} \ldots \partial_{3}^{\beta_{3}}$, where $\partial_{a}=\partial / \partial x^{a}$. We associate with the chosen Minkowski basis a Euclidean metric with unit matrix in that basis, and denote by $|x|$ the norm of $x$ in that metric. We briefly recall the definitions of test functions spaces used in [3]. Let $\phi(x)$ be a smooth tensor or spinor field (with vector representation of points) and define for $\kappa \geq 0, l=0,1, \ldots$ the seminorms

$$
\|\varphi\|_{\kappa, l}=\sup (1+|x|)^{\kappa}\left|D^{\beta} \varphi_{j}(x)\right|,
$$

where supremum is taken over $x \in M$, all $\beta$ such that $|\beta|=l$ and $j$ running over the components of the field. Then $\mathcal{S}_{\kappa}$ is the space of all smooth fields of a given geometrical type for which all seminorms $\|\cdot\|_{\kappa+l, l}$ with fixed $\kappa$ are finite. Denote moreover the operators on smooth functions $H=x \cdot \partial$ and $H_{\kappa}=H+\kappa$ id. Then the space $\mathcal{S}_{\kappa+\epsilon}^{\kappa}$ consists of all fields which under the action of $H_{\kappa}$ fall into $\mathcal{S}_{\kappa+\epsilon}$. Each field $\varphi \in \mathcal{S}_{\kappa+\epsilon}^{\kappa}$ has an asymptote

$$
\varphi_{\text {as }}(x)=\lim _{R \rightarrow \infty} R^{\kappa} \varphi(R x) .
$$

The inversion formulas are

$$
\varphi(x)=\int_{0}^{1} u^{\kappa-1}\left[H_{\kappa} \varphi\right](u x) \mathrm{d} u, \quad \varphi_{\mathrm{as}}(x)=\int_{0}^{\infty} u^{\kappa-1}\left[H_{\kappa} \varphi\right](u x) \mathrm{d} u .
$$

The subspaces $\mathcal{S}_{\kappa}(\Omega), \mathcal{S}_{\kappa+\epsilon}^{\kappa}(\Omega)$ consist of functions supported in $\Omega$. All spaces, as well as asymptotes, are independent of the choice of an origin and a basis. 
Next, we recall some notation for Lorentz invariant hypersurfaces. We denote by $l$ vectors on the future lightcone, and we also introduce

$$
L_{a b}=l_{a}\left(\partial / \partial l^{b}\right)-l_{b}\left(\partial / \partial l^{a}\right)
$$

which is an operator conveniently expressing differentiation on the lightcone. We denote by $\mathrm{d}^{2} l$ the invariant measure on the set of null directions, which is applicable to functions $f(l)$ homogeneous of degree -2 : the integral

$$
\int f(l) \mathrm{d}^{2} l=\int f\left(e_{0}+\vec{l}\right) \mathrm{d} \Omega(\vec{l}),
$$

where $\mathrm{d} \Omega(\vec{l})$ is the solid angle measure in the direction of the unit 3-vector $\vec{l}$, is independent of the choice of Minkowski basis, and satisfies

$$
\int L_{a b} f(l) \mathrm{d}^{2} l=0 .
$$

We denote by $H_{+}$the hyperboloid $v^{2}=1, v^{0}>0$. The differentiation within the hyperboloid is conveniently expressed by the action of the operator $\delta_{a}$, and integration with the use of invariant measure $\mathrm{d} \mu$, defined respectively by

$$
\delta_{b}=v^{a}\left[v_{a}\left(\partial / \partial v^{b}\right)-v_{b}\left(\partial / \partial v^{a}\right)\right], \quad \mathrm{d} \mu(v)=2 \theta\left(v^{0}\right) \delta\left(v^{2}-1\right) \mathrm{d}^{4} v .
$$

We note that for a differentiable function $f(v)$ vanishing for $v^{0} \rightarrow \infty$ as $o\left(\left(v^{0}\right)^{-3}\right)$, we have

$$
\int(\delta-3 v) f(v) \mathrm{d} \mu(v)=0
$$

For $x$ inside the future lightcone, one can write $x=\lambda v, \lambda>0$, and then differentiation and integration over the inside of the future lightcone may be written as

$$
\begin{aligned}
\partial / \partial x^{a} & =v_{a} \partial_{\lambda}+(1 / \lambda) \delta_{a} \\
\int F(x) \mathrm{d}^{4} x & =\int F(\lambda v) \lambda^{3} \mathrm{~d} \lambda \mathrm{d} \mu(v) .
\end{aligned}
$$

Similarly, for the hyperboloid $H_{-}$formed by $z^{2}=-1$, the differentiation operator and the integration measure are defined, respectively, by

$$
\delta_{b}=-z^{a}\left[z_{a}\left(\partial / \partial z^{b}\right)-z_{b}\left(\partial / \partial z^{a}\right)\right], \quad \mathrm{d} \nu(z)=2 \delta\left(z^{2}+1\right) \mathrm{d}^{4} z .
$$

For $f(z)$ vanishing for $|\vec{z}| \rightarrow \infty$ as $o\left(|\vec{z}|^{-3}\right)$, there is

$$
\int(\delta+3 z) f(z) \mathrm{d} \nu(z)=0
$$

and for $x=\lambda z(\lambda>0)$ running over the outside of the lightcone, the analogues of (2.7) and (2.8) are

$$
\begin{aligned}
\partial / \partial x^{a} & =-z_{a} \partial_{\lambda}+(1 / \lambda) \delta_{a} \\
\int F(x) \mathrm{d}^{4} x & =\int F(\lambda z) \lambda^{3} \mathrm{~d} \lambda \mathrm{d} \nu(z) .
\end{aligned}
$$


Finally, we define some spacetime sets used in the article. For $\gamma>0$ and $\delta \in(0,1)$ we shall denote by $R_{\gamma, \delta}$ the region $\left|x^{0}\right| \leq \gamma+\delta|\vec{x}|$ and by $R_{\delta}$ the region $\left|x^{0}\right| \leq \delta|\vec{x}|$. We note that

$$
-x^{2} \geq \frac{1-\delta^{2}}{1+\delta^{2}}|x|^{2} \quad \text { for } x \in R_{\delta} .
$$

By a spacelike cone, we shall mean a closed (solid) cone in $\mathcal{M}$ such that all vectors going from the apex to other points of the cone are spacelike. A symmetrical spacelike cone will be the union of such cone with its reflection with respect to its apex, and a fattened symmetrical spacelike cone - the union of such cone with its reflection with respect to a point inside the cone. An open version of any of the defined cones will be its interior.

\section{The Model}

We briefly summarize the model formulated in [1]. The choice of the test functions spaces is slightly modified.

\subsection{Electromagnetic Test Functions}

Let $V(s, l)$ be a real vector function of a real variable $s$ and a future-pointing lightlike vector $l$. We shall understand differentiability of functions $V_{a}$ in the sense of the action of $L_{a b}$ and $\partial_{s}=\partial / \partial s$, and denote $\dot{V}(s, l)=\partial_{s} V(s, l)$. Let $\mathcal{V}_{\epsilon}$ be the real vector space of $\mathcal{C}^{\infty}$ functions $V_{a}(s, l)$ which satisfy the following additional conditions:

$$
\begin{aligned}
V(\mu s, \mu l) & =\mu^{-1} V(s, l), \quad \mu>0, \\
l \cdot V(s, l) & =0, \\
\left|L_{b_{1} c_{1}} \ldots L_{b_{k} c_{k}} \dot{V}_{a}(s, l)\right| & \leq \frac{\operatorname{const}(t, k)}{(t \cdot l)^{2}(1+|s| / t \cdot l)^{1+\epsilon}}, \quad k \in \mathbb{N}, \\
V(+\infty, l) & =-V(-\infty, l) \equiv \frac{1}{2} \Delta V(l), \\
L_{[a b} \Delta V_{c]}(l) & =0,
\end{aligned}
$$

where the third condition holds for an arbitrarily chosen unit timelike, futurepointing vector $t$; the bounds are then true for any other such vector (with some other constants). Moreover, with the use of homogeneity (3.1), the bounds are generalized to

$$
\left|L_{b_{1} c_{1}} \ldots L_{b_{k} c_{k}} \partial_{s}^{n} V_{a}(s, l)\right| \leq \frac{\operatorname{const}(t, n, k)}{(t \cdot l)^{2}(1+|s| / t \cdot l)^{n+\epsilon}}, \quad n, k \in \mathbb{N},
$$

It follows from the property (3.5) that

$$
l_{a} \Delta V_{b}(l)-l_{b} \Delta V_{a}(l)=-L_{a b} \Phi_{V}(l),
$$

where

$$
\Phi_{V}(l)=-\frac{1}{4 \pi} \int \frac{l \cdot \Delta V\left(l^{\prime}\right)}{l \cdot l^{\prime}} \mathrm{d}^{2} l^{\prime}
$$


is a smooth homogeneous function. If $\Delta V(l)=l \alpha(l)$, then

$$
\Phi_{V}(l)=-\frac{1}{4 \pi} \int \alpha\left(l^{\prime}\right) \mathrm{d}^{2} l^{\prime}=\text { const. }
$$

We also note for later use that for $v \in H_{+}$, there is

$$
\int \frac{v \cdot \Delta V(l)}{v \cdot l} \mathrm{~d}^{2} l=-\int \frac{\Phi_{V}(l)}{(v \cdot l)^{2}} \mathrm{~d}^{2} l .
$$

The spaces $\mathcal{V}_{\epsilon}$ form an increasing family for $\epsilon \searrow 0$, so their union is a vector space,

$$
\mathcal{V}=\bigcup_{\epsilon>0} \mathcal{V}_{\epsilon}
$$

This vector space, when viewed as an Abelian group, allows the following suband quotient groups:

$$
\begin{gathered}
\mathcal{V}_{\text {as }}^{0}=\left\{V \in \mathcal{V} \mid l \wedge V(s, l)=0 \text { and } \Phi_{V}(l)=n(2 \pi / e), n \in \mathbb{Z}\right\}, \\
L=\mathcal{V} / \mathcal{V}_{\text {as }}^{0}
\end{gathered}
$$

the elements of the latter will be denoted by $[V]$. The space $\mathcal{V}$ is equipped with a symplectic form

$$
\left\{V_{1}, V_{2}\right\}=\frac{1}{4 \pi} \int\left(\dot{V}_{1} \cdot V_{2}-\dot{V}_{2} \cdot V_{1}\right)(s, l) \mathrm{d} s \mathrm{~d}^{2} l,
$$

which is also consistently transferred to $L$.

For each $V \in \mathcal{V}$, the formula

$$
A(x)=-\frac{1}{2 \pi} \int \dot{V}(x \cdot l, l) \mathrm{d}^{2} l
$$

gives the Lorentz potential of a free electromagnetic field with well-defined null asymptotes:

$$
\lim _{R \rightarrow \infty} R A(x \pm R l)= \pm V(x \cdot l, l)-\frac{1}{2} \Delta V(l)
$$

and a long-range tail of electric type. This is the class of fields which are produced in typical scattering processes [4]. For each spacelike $x$ and any fixed $a$, the spacelike tail is given by

$$
A^{\text {as }}(x)=\lim _{R \rightarrow \infty} R A(a+R x)=-\frac{1}{2 \pi} \int \Delta V(l) \delta(x \cdot l) \mathrm{d}^{2} l=A^{\text {as }}(-x),
$$

where $\delta$ is the Dirac measure. Let $F_{a b}^{\text {as }}$ be the electromagnetic field of this asymptotic potential. The condition (3.5) implies that $x_{[a} F_{b c]}^{\text {as }}(x)=0$, so this field is of electric type. If $F^{\text {as }}=0$, we shall say that the field is infrared-regular, otherwise it will be called infrared-singular. The symplectic form (3.13) is a natural extension, to the class considered here, of the usual symplectic form of free, infrared-regular electromagnetic fields. 


\subsection{Matter Test Functions}

We denote by $\mathcal{S}\left(H_{+}\right)$the space of smooth 4-spinor functions on $H_{+}$for which all seminorms

$$
\|f\|_{\alpha, \beta}^{H_{+}}=\sup \left|v^{\alpha} \delta^{\beta} f(v)\right|
$$

are finite (with the usual multi-index notation, and supremum over $v$ and components of the field).

For $f \in \mathcal{S}\left(H_{+}\right)$the Fourier representation in the form of the formula

$$
\psi(x)=\left(\frac{m}{2 \pi}\right)^{3 / 2} \int e^{-i m x \cdot v \gamma \cdot v} \gamma \cdot v f(v) \mathrm{d} \mu(v)
$$

gives a smooth Dirac field, with the timelike asymptote determined by

$$
f(v)=\lim _{\lambda \rightarrow \infty} \lambda^{3 / 2} i e^{i(m \lambda+\pi / 4) \gamma \cdot v} \psi(\lambda v) .
$$

One has the usual scalar product in the space of these fields

$$
\left(f_{1}, f_{2}\right)=\int \overline{f_{1}(v)} \gamma \cdot v f_{2}(v) \mathrm{d} \mu(v)=\int_{\Sigma} \overline{\psi_{1}} \gamma^{a} \psi_{2}(x) \mathrm{d} \sigma_{a}(x),
$$

where the second integral is over any Cauchy surface $\Sigma$. We denote by $\mathcal{K}$ the Hilbert space completion of $\mathcal{S}\left(H_{+}\right)$with respect to this product.

\subsection{The Algebra}

The ${ }^{*}$-algebra $\mathcal{B}$ of the model is generated by elements $W([V]),[V] \in L$, which for simplicity will also be written as $W(V)$, elements $\Psi(f), f \in \mathcal{S}\left(H_{+}\right)$, and a unit $E$ by

$$
\begin{aligned}
W\left(V_{1}\right) W\left(V_{2}\right) & =e^{-\frac{i}{2}\left\{V_{1}, V_{2}\right\}} W\left(V_{1}+V_{2}\right), \\
W(V)^{*} & =W(-V), W(0)=E, \\
{\left[\Psi\left(f_{1}\right), \Psi\left(f_{2}\right)\right]_{+} } & =0, \quad\left[\Psi\left(f_{1}\right), \Psi\left(f_{2}\right)^{*}\right]_{+}=\left(f_{1}, f_{2}\right) E, \\
W(V) \Psi(f) & =\Psi\left(S_{\Delta V} f\right) W(V),
\end{aligned}
$$

where

$$
\left(S_{\Delta V} f\right)(v)=\exp \left(-\frac{i e}{4 \pi} \int \frac{v \cdot \Delta V(l)}{v \cdot l} \mathrm{~d}^{2} l\right) f(v) .
$$

Note that the exponent function in the last formula is a multiplier in $\mathcal{S}\left(H_{+}\right)$, so the operator $S_{\Delta V}$ is a linear automorphism of $\mathcal{S}\left(H_{+}\right)$. This can be easily seen: since for $t \cdot l=1$ and $v \in H_{+}$there is $|v \cdot l|^{-1}<\left|v^{0}\right|+|\vec{v}|$, so $\left|\int \frac{\Delta V^{a}(l) l^{\alpha}}{(v \cdot l)^{|\alpha|+1}} \mathrm{~d}^{2} l\right|$ is polynomially bounded for any multi-index $\alpha$. Note also that, by the identity (3.9) and definitions (3.11) and (3.12), there is $S_{\Delta V_{2}}=S_{\Delta V_{1}}$ for $V_{2} \in\left[V_{1}\right] \in L$, so the algebra is properly defined.

The elements $\Psi(f)$ generate a subalgebra $\mathcal{B}^{+}$of the CAR type, and the elements $W(V)$ - a subalgebra $\mathcal{B}^{-}$of the CCR type. We denote by $\beta_{V}$ the automorphisms of $\mathcal{B}^{+}$defined by

$$
\beta_{V}(C)=W(V) C W(-V),
$$

forming a group, $\beta_{V_{1}} \beta_{V_{2}}=\beta_{V_{1}+V_{2}}$. 
Regular, translationally covariant, positive energy representations of $\mathcal{B}$ are shown, up to a unitary equivalence, to form a class defined in the following way. Let $\pi_{F}$ be the standard positive energy Fock representation of $\mathcal{B}^{+}$on $\mathcal{H}_{F}$ with the Fock vacuum vector $\Omega_{F}$, and $\pi_{r}$ be any regular, translationally covariant, positive energy representation of $\mathcal{B}^{-}$on $\mathcal{H}_{r}$. Define operators $\pi(A)$ on $\mathcal{H}=\mathcal{H}_{F} \otimes \mathcal{H}_{r}$ by

$$
\begin{aligned}
\pi(C) & =\pi_{F}(C) \otimes \mathrm{id}_{r}, \quad C \in \mathcal{B}_{\text {as }}^{+} \\
\pi(W(V))\left[\pi_{F}(B) \Omega_{F} \otimes \varphi\right] & =\pi_{F}\left(\beta_{V} B\right) \Omega_{F} \otimes \pi_{r}(W(V)) \varphi, \quad B \in \mathcal{B}_{\text {as }}^{+} .
\end{aligned}
$$

Then $\pi$ extends to a regular, translationally covariant positive energy representation of $\mathcal{B}$. We add one further demand to our selection criterion, that $\pi_{r}\left(W\left(V_{1}\right)\right)=\pi_{r}\left(W\left(V_{2}\right)\right)$ whenever $l \wedge V_{1}=l \wedge V_{2}$, which is related to the gauge invariance.

One shows that all representations from the class thus defined determine the same $C^{*}$-norm on $\mathcal{B}$; the completion of $\mathcal{B}$ in this norm is the $C^{*}$-algebra $\mathcal{F}$ of the model.

\section{Spacelike Localization of Electromagnetic Fields}

We now want to equip the elements of the algebra with spacetime localization properties. We start with the electromagnetic fields, which have direct observable status. The way to ascribe spacetime properties to them is to represent the classical test fields $A$ in (3.14) as

$$
A(x)=4 \pi \int D(x-y) J(y) \mathrm{d}^{4} y .
$$

Here $J$ is a classical conserved test current field, and $D(x)=D(0, x)$, with

$$
D(m, x)=\frac{i}{(2 \pi)^{3}} \int \operatorname{sgn} p^{0} \delta\left(p^{2}-m^{2}\right) e^{-i p \cdot x} \mathrm{~d} p .
$$

We want the supports of $J$ to be contained between two Cauchy surfaces. This may be interpreted as a generalized time-slice property.

We shall be concerned with conserved test currents $J$ which are elements of $\mathcal{S}_{3+\epsilon}^{3}\left(R_{\gamma, \delta}\right)$. Then the asymptote $J_{\text {as }}$ has the support in $R_{\delta}$. For such currents the integral in (4.1) is absolutely convergent and determines a corresponding $A$. We want to find out whether this potential is of the type given by (3.14). We start with a useful subsidiary result.

Lemma 1. Let $J_{\text {as }}$ be a homogeneous of degree -3 vector function, smooth outside the origin, with support in $R_{\delta}$. The following statements are equivalent.

(i) The continuity equation

$$
\partial \cdot J_{\mathrm{as}}(x)=0
$$

is satisfied distributionally. 
(ii) $J_{\text {as }}$ satisfies the following conditions on $\mathrm{H}_{-}$

$$
\begin{aligned}
\delta \cdot J_{\mathrm{as}}(z)+3 z \cdot J_{\mathrm{as}}(z) & =0, \\
\int z \cdot J_{\mathrm{as}}(z) \mathrm{d} \nu(z) & =0 .
\end{aligned}
$$

(iii) $J_{\mathrm{as}}$ is an asymptote of some conserved current $J \in \mathcal{S}_{3+\epsilon}^{3}\left(R_{\gamma, \delta}\right)$.

In particular, these conditions are satisfied for $J_{\text {as }}$ of the special form

$$
J_{\text {as }}(x)=x g(x) \text { with } \quad \int g(z) \mathrm{d} \nu(z)=0,
$$

where $g$ is a scalar function homogeneous of degree -4, smooth outside the origin.

Proof. The condition (4.4) is equivalent to (4.3) for $x$ outside the origin (use (2.10)). If it holds, then we have for any test function $\varphi$

$$
\begin{aligned}
\int J_{\text {as }}^{b}(x) \partial_{b} \varphi(x) \mathrm{d}^{4} x & =\lim _{\epsilon \rightarrow 0} \int_{x^{2}=-\epsilon^{2}} \varphi(x) J_{\text {as }}^{b}(x) \mathrm{d} \sigma_{b}(x) \\
& =\varphi(0) \int z \cdot J_{\text {as }}(z) \mathrm{d} \nu(z),
\end{aligned}
$$

which proves the equivalence of (i) and (ii). Let $\rho$ be a smooth function with support in $|x| \leq \gamma / \sqrt{2}$ and such that $\int \rho(x) \mathrm{d}^{4} x=1$. The vector function

$$
J_{\rho}=\rho * J_{\text {as }}
$$

is easily shown to be in $\mathcal{S}_{3+\epsilon}^{3}\left(R_{\gamma, \delta}\right)$ with the asymptote $J_{\text {as }}$, and if (i) is true, then it satisfies the continuity equation. Conversely, if $J_{\text {as }}$ is the asymptote of a conserved $J \in \mathcal{S}_{3+\epsilon}^{3}\left(R_{\gamma, \delta}\right)$, then it is supported in $R_{\delta}$ and (4.4) is the limit of the continuity equation $\partial \cdot J(x)=0$ for $x^{2} \rightarrow-\infty$. Integrating the latter equation over the region $x^{2} \geq-R^{2}$ and taking the limit $R \rightarrow \infty$ one arrives at (4.5). The statement concerning (4.6) is easily checked.

We note for future use that by (2.9) and (4.4) one has for any continuously differentiable function $f(z)$

$$
\int J_{\mathrm{as}} \cdot \delta f(z) \mathrm{d} \nu(z)=0
$$

Theorem 2. Let $J \in \mathcal{S}_{3+\epsilon}^{3}\left(R_{\gamma, \delta}\right)$ be a conserved current. Then the function

$$
\dot{V}(s, l)=\frac{1}{s}\left(V_{0}(s, l)-V_{0}(0, l)\right),
$$

where

$$
V_{0}(s, l)=\int \delta(s-x \cdot l) H_{3} J(x) \mathrm{d}^{4} x,
$$

satisfies conditions (3.1) and (3.2), and $J$ and $V$ generate the same $A$ according to (4.1) and (3.14) respectively. If the asymptote of $J$ is odd:

$$
J_{\text {as }}(-x)=-J_{\text {as }}(x),
$$


then $V_{0}(0, l)=0$, so $V$ satisfies also (3.3), and it may be then obtained by

$$
V(s, l)=\lim _{R \rightarrow \infty} V^{R}(s, l), \quad V^{R}(s, l)=\int_{x^{2} \geq-R^{2}} \delta(s-x \cdot l) J(x) \mathrm{d}^{4} x
$$

with $V^{R}(s, l)$ uniformly bounded and with

$$
\Delta V(l)=\int \frac{J_{\mathrm{as}}(z)}{z \cdot l} \mathrm{~d} \nu(z)
$$

(the integral in the principal value sense). If in addition $L_{[a b} \Delta V_{c]}(l)=0$, then $V \in \mathcal{V}_{\epsilon}$. This is, in particular, fulfilled for $J_{\text {as }}$ of the type given by (4.6) with even $g(z)$.

If $J_{1}$ and $J_{2}$ are two currents satisfying all the above assumptions, then

$$
\left\{V_{1}, V_{2}\right\}=\lim _{R \rightarrow \infty} \frac{1}{2} \int_{x^{2} \geq-R^{2}}\left[J_{1} \cdot A_{2}-J_{2} \cdot A_{2}\right](x) \mathrm{d}^{4} x .
$$

Proof. We first observe that as $H_{3} J(x)$ vanishes as $|x|^{-3-\epsilon}$ in infinity, the integral (4.11) is absolutely convergent, and relations (3.1) and (3.2) are easily seen to hold for $V_{0}$. Moreover, with $X_{a b}=x_{a} \partial / \partial x^{b}-x_{b} \partial / \partial x^{a}$, we have

$$
\begin{aligned}
& \left|L_{a_{1} b_{1}} \ldots L_{a_{k} b_{k}} V_{0}(s, l)\right|=\left|\int \delta(s-x \cdot l) X_{a_{1} b_{1}} \ldots X_{a_{k} b_{k}} H_{3} J(x) \mathrm{d}^{4} x\right| \\
& \quad \leq \text { const } \int \delta(s-x \cdot l)(1+|x|)^{-3-\epsilon} \mathrm{d}^{4} x \leq \frac{\mathrm{const}}{t \cdot l(1+|s| / t \cdot l)^{\epsilon}}
\end{aligned}
$$

If $A$ is generated by $J$, then one finds easily that $H_{1} A$ is generated by $H_{3} J$. It is then also easily seen, using the representation

$$
D(x)=-\left(1 / 8 \pi^{2}\right) \int \delta^{\prime}(x \cdot l) \mathrm{d}^{2} l,
$$

that $\dot{V}_{0}$ generates $H_{1} A$ by (3.14). But then it follows that $A$ may be obtained by (3.14) from $\dot{V}$ defined by (4.10).

We want to obtain another form of $V_{0}$. For any $R>0$ we have

$$
\begin{aligned}
\partial \cdot\{ & \left.x \delta(s-x \cdot l)\left[J(x)-\theta\left(-x^{2}-R^{2}\right) J_{\mathrm{as}}(x)\right]\right\} \\
= & -s \delta^{\prime}(s-x \cdot l)\left[J(x)-\theta\left(-x^{2}-R^{2}\right) J_{\mathrm{as}}(x)\right] \\
& \quad+\delta(s-x \cdot l) H_{3} J(x)-2 R^{2} \delta(s-x \cdot l) \delta\left(x^{2}+R^{2}\right) J_{\mathrm{as}}(x) .
\end{aligned}
$$

The l.h.s. yields zero when integrated over whole space, so we find

$$
\begin{aligned}
V_{0}(s, l)= & s \partial_{s} \int \delta(s-x \cdot l)\left[J(x)-\theta\left(-x^{2}-R^{2}\right) J_{\text {as }}(x)\right] \mathrm{d}^{4} x \\
& +\int \delta\left(\frac{s}{R}-z \cdot l\right) J_{\text {as }}(z) \mathrm{d} \nu(z) .
\end{aligned}
$$

Setting here $s=0$, we find

$$
V_{0}(0, l)=\int \delta(z \cdot l) J_{\mathrm{as}}(z) \mathrm{d} \nu(z),
$$


so if $J_{\text {as }}$ is odd, what we assume from now on, there is $V_{0}(0, l)=0$, and then $V$ satisfies the bounds (3.3) (use (4.16)). We note that if $V_{0}(0, l) \neq 0$, then $\dot{V}(s, l)$ falls off only as $1 /|s|$ and is outside the class $\mathcal{V}$.

We integrate (4.10) with the use of (4.18), and find

$$
\begin{aligned}
V(s, l)-V(-\infty, l)= & V^{R}(s, l)+\int_{-\infty}^{s / R} \frac{1}{\tau}\left\{\int \delta(\tau-z \cdot l) J_{\text {as }}(z) \mathrm{d} \nu(z)\right\} \mathrm{d} \tau \\
& +\int_{x \leq-R^{2}} \delta(s-x \cdot l)\left(J-J_{\text {as }}\right)(x) \mathrm{d}^{4} x
\end{aligned}
$$

with $V^{R}$ as defined in (4.13). The last term vanishes both in the limit $R \rightarrow \infty$ as well as $|s| \rightarrow \infty$, and $V^{R}(s, l)$ vanishes for $|s| \rightarrow \infty$; the uniform boundedness of $V^{R}(s, l)$ is also easily seen. We write down the limit versions of (4.20) for $R \rightarrow \infty$ and for $s \rightarrow \infty$, respectively (remember that $V(+\infty, l)=$ $\left.-V(-\infty, l)=\frac{1}{2} \Delta V(l)\right)$

$$
\begin{gathered}
V(s, l)+\frac{1}{2} \Delta V(l)=\lim _{R \rightarrow \infty} V^{R}(s, l)+\int_{-\infty}^{0} \frac{1}{\tau}\left\{\int \delta(\tau-z \cdot l) J_{\text {as }}(z) \mathrm{d} \nu(z)\right\} \mathrm{d} \tau, \\
\Delta V(l)=\int_{-\infty}^{+\infty} \frac{1}{\tau}\left\{\int \delta(\tau-z \cdot l) J_{\mathrm{as}}(z) \mathrm{d} \nu(z)\right\} \mathrm{d} \tau=\lim _{\epsilon \rightarrow 0} \int_{|z \cdot l| \geq \epsilon} \frac{J_{\mathrm{as}}(z)}{z \cdot l} \mathrm{~d} \nu(z) .
\end{gathered}
$$

The last equation gives (4.14). Owing to the oddness of $J_{\text {as }}$ the second term on the r.h.s. of (4.21) is then $\frac{1}{2} \Delta V(l)$, and we thus obtain (4.13). If (3.5) is satisfied, then $V \in \mathcal{V}_{\epsilon}$. We note that the differentiation on the cone is transferred to the differentiation on the hyperboloid:

$$
L_{a b} \int \frac{J_{\mathrm{as}}(z)}{z \cdot l} \mathrm{~d} \nu(z)=\int \frac{\left(z_{a} \delta_{b}-z_{b} \delta_{a}\right) J_{\mathrm{as}}(z)}{z \cdot l} \mathrm{~d} \nu(z),
$$

therefore $\Delta V$ is smooth, and for $J_{\text {as }}=z g(z)$ the condition (3.5) is satisfied automatically.

The last point concerns the symplectic form. We have

$$
\begin{aligned}
& \frac{1}{4 \pi} \int\left(\dot{V}_{1} \cdot V_{2}^{R}-\dot{V}_{2} \cdot V_{1}^{R}\right)(s, l) \mathrm{d} s \mathrm{~d}^{2} l \\
& =\frac{1}{4 \pi} \int_{x^{2} \geq-R^{2}}\left[\dot{V}_{1}(x \cdot l) J_{2}(x)-\dot{V}_{2}(x \cdot l) \cdot J_{1}(x)\right] \mathrm{d}^{2} l \mathrm{~d}^{4} x \\
& =\frac{1}{2} \int_{x^{2} \geq-R^{2}}\left(J_{1} \cdot A_{2}-J_{2} \cdot A_{1}\right)(x) \mathrm{d}^{4} x
\end{aligned}
$$

due to the representation (3.14). As $V_{i}^{R}(s, l)$ are uniformly bounded, by the Lebesgue theorem the 1.h.s. has a finite limit $\left\{V_{1}, V_{2}\right\}$ for $R \rightarrow \infty$, so also 
the r.h.s. has a finite limit, and one arrives at (4.15). However, we note that the integrand of the r.h.s. is not absolutely integrable on the whole space. The mechanism of the convergence in the limit relays on the fact that the asymptotes of $J_{i}$ are odd, while those of $A_{i}$ are even, so their products do not contribute, if integration is done in the above sense.

A particular test current $J_{\rho} \in \mathcal{S}_{3+\epsilon}^{3}\left(R_{\gamma, \delta}\right)$ with the given asymptote $J_{\text {as }}$ supported in $R_{\delta}$ was given in (4.8). We want to find its corresponding function $V_{\rho}$. We start with the following geometrical observation: for $y \in R_{\delta}$ and $|x-y| \leq \gamma$ there is

$$
\left|\theta\left(x^{2}+R^{2}\right)-\theta\left(y^{2}+R^{2}\right)\right| \leq \theta\left(-y^{2}-\left(R-R_{1}\right)^{2}\right) \theta\left(y^{2}+\left(R+R_{2}\right)^{2}\right)
$$

for $R \geq R_{1}$, with some $\gamma$ - and $\delta$-dependent constants $R_{1}, R_{2}$. This seems rather intuitive, but we give a formal proof in Appendix. It is then easy to see that instead of formula (4.13) one can use $V_{\rho}=\lim _{R \rightarrow \infty} V_{\rho}^{\prime} R$ with

$$
V_{\rho}^{\prime} R(s, l)=\int \delta(s-w \cdot l-y \cdot l) \rho(w) \theta\left(y^{2}+R^{2}\right) J_{\text {as }}(y) \mathrm{d}^{4} w \mathrm{~d}^{4} y .
$$

If we denote

$$
\begin{aligned}
H(s, l) & =\int \operatorname{sgn}(s-x \cdot l) \rho(x) \mathrm{d}^{4} x, \\
V_{\text {as }}^{R}(s, l) & =\int \delta(s-x \cdot l) \theta\left(x^{2}+R^{2}\right) J_{\text {as }}(x) \mathrm{d}^{4} x,
\end{aligned}
$$

then we have

$$
V_{\rho}^{\prime} R(s, l)=\frac{1}{2} \int \dot{H}(s-\tau, l) V_{\mathrm{as}}^{R}(\tau, l) \mathrm{d} \tau .
$$

Using in the following first step (2.11) and the homogeneity of $J_{\text {as }}(x)$, and in the second step oddness of $J_{\text {as }}(x)$, we find

$$
\begin{aligned}
V_{\mathrm{as}}^{R}(\tau, l) & =\int \theta\left(\frac{\tau}{z \cdot l}\right) \theta\left(R-\frac{\tau}{z \cdot l}\right) \frac{J_{\mathrm{as}}(z)}{|z \cdot l|} \mathrm{d} \nu(z) \\
& =\frac{1}{2} \operatorname{sgn}(\tau) \int_{|z \cdot l| \geq \frac{|\tau|}{R}} \frac{J_{\mathrm{as}}(z)}{z \cdot l} \mathrm{~d} \nu(z) .
\end{aligned}
$$

Thus for $R \rightarrow \infty$ the absolute value of (4.29) remains bounded, and one finds

$$
V_{\rho}(s, l)=H(s, l) \frac{1}{2} \Delta V(l),
$$

with $\Delta V(l)$ given by (4.14). Note that $H( \pm \infty, l)= \pm 1$.

Assume now that $\Delta V(l)$ satisfies (3.5) and is therefore determined up to a gauge by $\Phi_{V}(l)$.

Proposition 3. For $\Delta V(l)$ given by (4.14) there is

$$
\Phi_{V}(l)=\int z \cdot J_{\mathrm{as}}(z) \log |z \cdot l| \mathrm{d} \nu(z)
$$


Proof. We observe that the formula (3.8) defines in fact a continuous, homogeneous function $\Phi_{V}(x)$ for $x$ in the closed future lightcone. For $x$ inside the cone, and with $v=x / \sqrt{x^{2}}$, one finds

$$
\Phi_{V}(x)=-\int \frac{v \cdot J_{\mathrm{as}}(z)}{\sqrt{(v \cdot z)^{2}+1}} \log \left[\sqrt{(v \cdot z)^{2}+1}+v \cdot z\right] \mathrm{d} \nu(z),
$$

where we used the following formula valid for $v^{2}=1, v^{0}>0$ and $z^{2}=-1$ :

$$
\int \frac{\mathrm{d}^{2} l}{v \cdot l z \cdot l}=\frac{4 \pi}{\sqrt{(v \cdot z)^{2}+1}} \log \left[\sqrt{(v \cdot z)^{2}+1}+v \cdot z\right] .
$$

We observe that $\delta_{a}^{(z)}(v \cdot z)=v_{a}+v \cdot z z_{a}$, which allows us to write the integrand in (4.33) as

$$
\begin{aligned}
& \frac{1}{2} J_{\mathrm{as}}(z) \cdot \delta\left(\log \left[\sqrt{(v \cdot z)^{2}+1}+v \cdot z\right]\right)^{2} \\
& \quad+z \cdot J_{\mathrm{as}}(z)\left[1-\frac{|v \cdot z|}{\sqrt{(v \cdot z)^{2}+1}}\right] \log \left[\sqrt{(v \cdot z)^{2}+1}+|v \cdot z|\right] \\
& \quad-z \cdot J_{\mathrm{as}}(z) \log \frac{1}{2}\left[\sqrt{(x \cdot z)^{2}+x^{2}}+|x \cdot z|\right]+z \cdot J_{\mathrm{as}}(z) \log \frac{1}{2} \sqrt{x^{2}}
\end{aligned}
$$

where we used the fact that $\xi \log \left[\sqrt{\xi^{2}+1}+\xi\right]=|\xi| \log \left[\sqrt{\xi^{2}+1}+|\xi|\right]$. The first and the last terms give no contribution to the integral (use (4.9) and (4.5) respectively). We consider the other terms in the limit $x \rightarrow l$. In this limit $|v \cdot z|$ tends to $+\infty$ almost everywhere, and the second term remains bounded by const $\left|z \cdot J_{\text {as }}(z)\right|$ and tends to zero almost everywhere, so the contribution to the integral vanishes in this limit. Finally, the third term gives the thesis.

The above result has an interesting consequence.

Proposition 4. Let $J \in \mathcal{S}_{3+\epsilon}^{3}\left(R_{\gamma, \delta}\right)$ be a conserved current with an odd asymptote $J_{\mathrm{as}}$ and the corresponding function $V(s, l)$. Let $L_{[a b} \Delta V_{c]}(l)=0$, so that $V \in \mathcal{V}_{\epsilon}$.

Then there exists a current $J^{\prime}$ of the same type, but whose asymptote is of the particular form $J_{\text {as }}^{\prime}(z)=z g(z)$, such that the corresponding function $V^{\prime}(s, l)$ satisfies

$$
l \wedge\left(V^{\prime}-V\right)(s, l)=0 \quad \text { and } \quad \Phi_{V^{\prime}}(l)=\Phi_{V}(l) .
$$

Thus, in particular, $V^{\prime}(s, l)-V(s, l) \in \mathcal{V}_{\mathrm{as}}^{0}$ and $\left[V^{\prime}\right]=[V] \in L$.

Proof. We set $J^{\prime}=J+\rho *\left(J_{\text {as }}^{\prime}-J_{\text {as }}\right)$, where $J_{\text {as }}^{\prime}$ is homogeneous of degree -3 and on the unit hyperboloid given by $J_{\text {as }}^{\prime}(z)=-z z \cdot J_{\text {as }}(z)$, which then indeed is the asymptote of $J^{\prime}$. Then by (4.31) there is

$$
\left(V^{\prime}-V\right)(s, l)=H(s, l) \frac{1}{2}\left(\Delta V^{\prime}-\Delta V\right)(l)
$$

and by Proposition 3: $\Phi_{V^{\prime}}(l)=\Phi_{V}(l)$. Therefore $l \wedge\left(\Delta V^{\prime}-\Delta V\right)(l)=0$, which completes the proof. 
The net result of the present section to this point is the identification of a class of currents giving rise to test elements $[V] \in L$ of our electromagnetic Weyl algebra. Now we want to show that the whole group $L$ is covered in this way, and even more, that the class may be still narrowed. We start with an auxiliary result.

Lemma 5. Let a smooth function $W(s, l)$ be homogeneous of degree $n-$ $2, W(\mu s, \mu l)=\mu^{n-2} W(s, l)(\mu>0)$, and satisfy the falloff conditions

$$
\left|L_{b_{1} c_{1}} \ldots L_{b_{k} c_{k}} W(s, l)\right| \leq \operatorname{const}(k) \frac{(t \cdot l)^{n-2}}{(1+|s| / t \cdot l)^{\epsilon}}, \quad k \in \mathbb{N} .
$$

Denote $W^{(k)}(s, l)=\partial_{s}^{k} W(s, l)$ and set

$$
K(x)=-\frac{1}{2 \pi} \int W^{(n)}(x \cdot l, l) \mathrm{d}^{2} l .
$$

Then for each fixed $\delta \in(0,1)$ one has in the region $R_{\delta}$ the bounds

$$
|K(a+x)| \leq \operatorname{const}(\delta)(1+|x|)^{-n-\epsilon} .
$$

Proof. It is sufficient to show this for $a=0$, as the properties of $W$ are conserved under translations. For $n=0$ and $x \in R_{\delta}$, we have

$$
|K(x)| \leq \mathrm{const} \int_{-1}^{1} \frac{\mathrm{d} u}{\left(1+|| x^{0}|+| \vec{x}|u|\right)^{\epsilon}} \leq \operatorname{const}(\delta)(1+|x|)^{-\epsilon},
$$

We proceed by induction with respect to $n$. If we denote

$$
\tilde{x}(t, l)=(t \cdot l)^{-1} x+(t \cdot l)^{-2} t \cdot x l,
$$

then we have the identity

$$
\begin{aligned}
& L_{a b}\left[t^{a} \tilde{x}^{b} W^{(n-1)}(x \cdot l, l)\right] \\
& \quad=x^{2} W^{(n)}(x \cdot l, l)+\left[t^{a} \tilde{x}^{b} L_{a b}^{\prime}+\frac{x \cdot l}{(t \cdot l)^{2}}\right] W^{(n-1)}(x \cdot l, l),
\end{aligned}
$$

where $L_{a b}^{\prime} W^{(n-1)}(x \cdot l, l)=\left.L_{a b} W^{(n-1)}(s, l)\right|_{s=x \cdot l}$. The integral of the 1.h.s. over $l$ vanishes, so by induction we have

$$
\begin{aligned}
|K(x)| & \leq \min \left\{\operatorname{const}, \operatorname{const}(\delta) \frac{|x|}{\left|x^{2}\right|}(1+|x|)^{-n+1-\epsilon}\right\} \\
& \leq \operatorname{const}(\delta)(1+|x|)^{-n-\epsilon} .
\end{aligned}
$$

We can now prove our main result of this subsection.

Theorem 6. Let $A$ be given by the formula (3.14) with $V \in \mathcal{V}_{\epsilon}$, and chose an arbitrary set of the type $R_{\gamma, \delta}$. Then:

(i) There exists $V^{\prime} \in \mathcal{V}_{\epsilon}$ such that $\left[V^{\prime}\right]=[V]$ and the corresponding potential $A^{\prime}$ may be represented as a radiation potential of a test current $J^{\prime} \in \mathcal{S}_{3+\epsilon}^{3}\left(R_{\gamma, \delta}\right)$ with the asymptote of the form $J_{\text {as }}^{\prime}(x)=x \rho(x)$, with $\rho(-x)=\rho(x)$, supported in $R_{\delta}$. 
(ii) The test current $J^{\prime}$ may be represented as a sum of currents with the same properties, but in addition each of the currents is supported in a fattened symmetrical spacelike cone contained in $R_{\gamma, \delta}$. For each cover of the set $R_{\gamma, \delta}$ with such cones there is a corresponding split of $J^{\prime}$.

Proof. For a given $A$ and $V$, we define

$$
C^{a}(x)=-\frac{1}{2 \pi} \int \frac{V^{a}(x \cdot l, l)}{t \cdot l} \mathrm{~d}^{2} l, \quad B^{a b}=C^{a} t^{b}-C^{b} t^{a} .
$$

Then $\square B^{a b}(x)=0$ and $A^{a}(x)=\partial_{b} B^{a b}(x)$. Moreover, with the use of the above lemma one finds easily that for $x \in R_{\delta}$, there is

$$
\left|D^{\alpha} H_{0} C(a+x)\right| \leq \operatorname{const}(a, \delta, \alpha)(1+|x|)^{-|\alpha|-\epsilon} .
$$

Let now $F$ be a smooth function on the spacetime which for $|x| \geq \gamma$, for some $\gamma>0$, satisfies:

(i) $F(\mu x)=F(x)$ for all $\mu \geq 1$ (homogeneity),

(ii) $F(-x)=-F(x)$,

(iii) $F(x)=1 / 2$ for $x^{0} \geq \delta|\vec{x}|$ for some $\delta \in(0,1)$.

Note that the supports of derivatives of $F$ are contained in $R_{\gamma, \delta}$. We claim that

$$
B^{a b}(x)=4 \pi \int D(x-y) \varphi^{a b}(y) \mathrm{d}^{4} y,
$$

where $\varphi^{a b}(y)=\square\left(F(y) B^{a b}(y)\right)$. Indeed, the support of $\varphi$ is contained in $R_{\gamma, \delta}$, and for $x$ in the future of $R_{\gamma, \delta}$ the r.h.s. may be written as

$$
4 \pi \int D_{\text {ret }}(x-y) \square\left(\left[F(y)+\frac{1}{2}\right] B^{a b}(y)\right) \mathrm{d}^{4} y,
$$

which yields the l.h.s. upon integration by parts. But both sides satisfy the wave equation, so the equality holds everywhere.

The fall-off properties (4.44) now easily imply that $\varphi \in \mathcal{S}_{2+\epsilon}^{2}$. Moreover, the support of $\varphi$ is contained in $R_{\gamma, \delta}$ and that of the asymptote $\varphi_{\text {as }}$ in $R_{\delta}$, and the asymptote is even: $\varphi_{\text {as }}(-x)=\varphi_{\text {as }}(x)$. The potential $A$ has now the representation (4.1) with the test current $J^{a}=\partial_{b} \varphi^{a b}$, which is an element of $S_{3+\epsilon}^{3}$, has similar support properties as $\varphi$, and its asymptote is odd: $J_{\text {as }}(-x)=-J_{\text {as }}(x)$. Thus $J$ satisfies all the assumptions of the Proposition 4. The current $J^{\prime}$ defined in the proof of this proposition may be written in the present case as $J^{\prime}=J_{\text {reg }}^{\prime}+J_{\text {sing }}^{\prime}$ with

$$
\begin{gathered}
J_{\text {reg }}^{\prime a}=\partial_{b} \varphi_{\text {reg }}^{a b}, \quad \varphi_{\text {reg }}=\varphi-\rho * \varphi_{\text {as }}, \\
J_{\text {sing }}^{\prime}=\rho * J_{\text {as }}^{\prime}, \quad J_{\text {as }}^{\prime}(x)=x\left(\frac{x_{c} \partial_{b} \varphi_{\text {as }}^{c b}(x)}{x^{2}}\right) .
\end{gathered}
$$

This completes the proof of (i).

To show (ii), we apply the above construction to $R_{\gamma^{\prime}, \delta^{\prime}}$ with $\gamma^{\prime}<\gamma, \delta^{\prime}<\delta$, and note that the two parts $J_{\text {reg }}^{\prime}$ and $J_{\text {sing }}^{\prime}$ may be considered separately. For the first part we note a rather obvious fact: for each cover of $R_{\gamma^{\prime}, \delta^{\prime}}$ with open 
fattened symmetrical spacelike cones contained in $R_{\gamma, \delta}$ there exist a decomposition of unity on $R_{\gamma^{\prime}, \delta^{\prime}}$ with smooth functions $f_{k}$ supported in the respective fattened symmetrical cones, taking values in $\langle 0,1\rangle$ and with bounded all derivatives. The currents $J_{\text {reg, }, k}^{\prime a}=\partial_{b}\left(f_{k} \varphi_{\text {reg }}^{a b}\right)$ satisfy the thesis. For the second part we note that the intersection of $H_{-}$with $R_{\delta^{\prime}}$ may be covered by arbitrarily small symmetrical patches, which are open as subsets of $H_{-}$and are contained in $R_{\delta}$. For each such cover there exists a corresponding decomposition of unity on $R_{\delta^{\prime}} \cap H_{-}$with smooth, even functions $g_{k}(z)$ supported in the respective patches, taking values in $\langle 0,1\rangle$ and with bounded derivatives. We extend these functions by homogeneity and define

$$
J_{\text {sing }, k}^{\prime}=\rho * J_{\mathrm{as}, k}^{\prime}, \quad J_{\mathrm{as}, k}^{\prime}(x)=x\left(\frac{x_{c} \partial_{b} \varphi_{\mathrm{as}, k}^{c b}(x)}{x^{2}}\right), \quad \varphi_{\mathrm{as}, k}^{a b}=g_{k} \varphi_{\mathrm{as}}^{a b} .
$$

The asymptotes $J_{\text {as }, k}^{\prime}$ are odd and satisfy

$$
\int z \cdot J_{\mathrm{as}, k}^{\prime}(z) \mathrm{d} \nu(z)=\int \delta_{b}\left(z_{c} \varphi_{\mathrm{as}, k}^{c b}(z)\right) \mathrm{d} \nu(z)=0
$$

by $(2.9)$, so $J_{\text {sing, }, k}^{\prime}$ are conserved currents by (4.5). Their sum yields $J_{\text {sing }}^{\prime}$, which ends the proof.

\section{Localization of Dirac Fields and Observables}

Fields carrying charge do not represent observables. Even more, in full electrodynamics they undergo local gauge transformations, thus to form an observable with the use of them one has to compensate not only the global, but also local gauge scaling. If $\Psi(x)$ and $A(x)$ represent 'local quantum spacetime fields', then a way to achieve this is to give a precise meaning (by smearing, renormalization etc.) to the heuristically formed quantities $\bar{\Psi}(x) \exp \left(-i e \int_{x}^{y} A(z) \mathrm{d} z\right) \Psi(y)$. Localization of this quantity, if it can be defined, should be determined by spacetime points $x$ and $y$ and the integration path between them.

Single fields creating or annihilating a physical charged particle, on the other hand, interpolate between different representations of observables. However, because of the Gauss law they cannot be local. Staying at the adopted heuristic level, the best that one can do is to cut the above quantity in two and obtain $\exp \left(-i e \int_{\infty}^{y} A(z) \mathrm{d} z\right) \Psi(y)$, where the path goes to spacelike infinity. The expectation then would be that the effect of this operation is invisible in the region spacelike to the localization of the integration path.

The above naive picture has its more refined counterpart in the algebraic analysis of the superselection sectors in quantum electrodynamics made by Buchholz [5]. The idea behind the selection criterion adopted in this analysis is that by an appropriate choice of the 'radiation cloud' superimposed on a charged state one can concentrate at a given time the electric flux at spacelike infinity in an arbitrarily chosen patch on the 2-sphere in the infinity of 3-space. The causal influence of the presence of the charge in this state may be thus 
made to vanish in the causal complement of some spacelike cone in Minkowski space.

We shall now investigate this question in the model defined here. Our algebra is an algebra of fields, not only observables, thus we formulate the problem in their terms. We shall ask whether, in representations defined in Sect. 3.3, by composing the charged field $\pi(\Psi(f))$ with some radiation cloud and a subsequent rescaling (to push the cloud to spacelike infinity), one can obtain a modified field restricted to a fattened symmetrical spacelike cone. The infrared tails are symmetric in the class of fields considered in the model, thus the replacement of spacelike cones by fattened symmetrical spacelike cones is unavoidable.

We shall see that the answer to this question is negative for a rather general construction reflecting in an obvious way the above idea. This seems to disagree also with expectations based on perturbative calculations in QED. The 'perturbative axiomatic' construction of the physical state space by Steinmann [6] may be seen as the strongest indication in this direction. We postpone the discussion of this point to the concluding section.

On the other hand, the same construction will allow us to construct local observables formed as products of 'dressed' Dirac fields and their adjoints.

\subsection{Spacelike Test Functions}

To ascribe localization to elements $\Psi(f)$, we first have to interpret test functions in spacetime terms; this will be done in this subsection. However, this will not give the full answer to the question because of noncommutativity with observables $W(V)$. We treat then the addition of the clouds in further subsections.

The first step is achieved, in analogy to the electromagnetic case, by representing the classical test field $\psi$ in (3.18) as

$$
\psi(x)=\frac{1}{i} \int S(m, x-y) \chi(y) \mathrm{d}^{4} y
$$

where $\chi$ is a classical test 4-spinor field and $S(m, x)=(i \gamma \cdot \partial+m) D(m, x)$. We want the support $\chi$ to be contained between two Cauchy surfaces.

It is easy to show that the Fourier representation of $S(m, x)$ may be written as

$$
S(m, x)=i\left(\frac{m}{2 \pi}\right)^{3} \int e^{-i m x \cdot v \gamma \cdot v} \gamma \cdot v \mathrm{~d} \mu(v)
$$

and then the Fourier connection between $f(v)$ and $\chi(x)$ in the integral representations of the Dirac field $\psi$ given respectively by (3.18) and (5.1) takes the form

$$
f(v)=\left(\frac{m}{2 \pi}\right)^{3 / 2} \int e^{i m v \cdot x \gamma \cdot v} \chi(x) \mathrm{d}^{4} x .
$$

It is clear that if $\chi \in \mathcal{S}(\mathcal{M})$, the Schwartz functions space, then $f \in \mathcal{S}\left(H_{+}\right)$. For the converse statement we note first the following analogue of the 'regular wave packet' property. 
Proposition 7. If $f \in \mathcal{S}\left(H_{+}\right)$, then for each $\delta \in(0,1)$ the Dirac field $\psi$ formed by (3.18) satisfies in the region $R_{\delta}$ the bounds

$$
\left|D^{\beta} \psi(x)\right| \leq \operatorname{const}(\delta,|\beta|, n)(1+|x|)^{-n}
$$

for each $\beta$ and each $n \in \mathbb{N}$.

Proof. The representation (3.18) is proportional to the sum of two terms $\int e^{\mp i m v \cdot x} f_{ \pm}(v) \mathrm{d} \mu(v)$ with $f_{ \pm}=P_{ \pm}(v) f(v), P_{ \pm}(v)=\frac{1}{2}(1 \pm \gamma \cdot v)$. It is clear that application of $D^{\beta}$ only modifies functions $f_{ \pm}$. Now, for any $g \in \mathcal{S}\left(H_{+}\right)$ and $x^{2}<0$, we have the identity

$$
\begin{aligned}
& \int e^{ \pm i m v \cdot x} g(v) \mathrm{d} \mu(v) \\
& =\left(\frac{ \pm i}{m}\right)^{n} \int \frac{e^{ \pm i m v \cdot x}}{\left[x^{2}-(v \cdot x)^{2}\right]^{n}}\left[\prod_{k=1}^{n} x \cdot(\delta+(2 k-3) v)\right] g(v) \mathrm{d} \mu(v),
\end{aligned}
$$

where the operators under the product sign are ordered from right to left with increasing $k$. This is easily shown by induction with respect to $n$ (integrate the r.h.s. by parts with the use of (2.6)). But using (2.12), we have $\left|x^{2}-(v \cdot x)^{2}\right| \geq \operatorname{const}(\delta)|x|^{2}$ for $x \in R_{\delta}$. This leads easily to the thesis.

Theorem 8. Let $\psi$ be given by the formula (3.18) with $f \in \mathcal{S}\left(H_{+}\right)$, and chose an arbitrary set of the type $R_{\gamma, \delta}$ (here $\delta=0$ is also admitted). Then there exists $\chi \in \mathcal{S}\left(R_{\gamma, \delta}\right)$ which generates $\psi$ by (5.1) (and, therefore, generates $f$ by $(5.3))$.

Proof. Let $F$ be the function defined in the proof of Theorem 6, and set $\chi=(\gamma \cdot \partial+i m)(F \psi)$. This function has support in $R_{\gamma, \delta}$, and with the use of the last proposition one then easily shows that it is a Schwartz function. Using the method employed in the proof of Theorem 6, one finds that $\chi$ generates $\psi$.

\section{2. 'Dressed' Charged Fields}

We now want to add radiation clouds to the Dirac fields. We first treat the problem heuristically, and write the Dirac field in the 'integrational' notation as $\Psi(f)=\int \overline{f(v)} \gamma \cdot v \Psi(v) \mathrm{d} \mu(v)$. For each four-velocity of the particle $v$ we choose an electromagnetic cloud profile $V_{v}(s, l) \in \mathcal{V}$, and form a modified field $\Psi\left(f, V_{*}\right)=\int \overline{f(v)} \gamma \cdot v W\left(V_{v}\right) \Psi(v) \mathrm{d} \mu(v)$. This, of course, has only a heuristic value, but one can expect that this field can be constructed in the von Neumann algebra of a representation (from the class defining the $C^{*}$-algebra $\mathcal{F}$ ). Let us write, still at this informal level, the commutation relation of this field with the electromagnetic field. We find

$$
W\left(V_{1}\right) \Psi\left(f, V_{*}\right)=\Psi\left(S_{V_{1}, V_{*}} f, V_{*}\right) W\left(V_{1}\right),
$$

where $\left(S_{V_{1}, V_{*}} f\right)(v)=\exp \left[i \varphi_{V_{1}, V_{*}}(v)\right] f(v)$ with

$$
\varphi_{V_{1}, V_{*}}(v)=-\frac{e}{4 \pi} \int \frac{v \cdot \Delta V_{1}(l)}{v \cdot l} \mathrm{~d}^{2} l+\left\{V_{1}, V_{v}\right\} .
$$


The problem of compensating the Coulomb field by the cloud field in some region is now the problem of choosing $V_{v}$ so as to compensate the first term in (5.7) by the second term, for $V_{1}$ in some class. However, we note that the symplectic form reduces to zero when restricted to any of the two subspaces of functions $V(s, l)$ which are even or odd in $s$ respectively. But $\Delta V_{1}(l)$ is the characteristic of the odd part of $V_{1}(s, l)$. Thus the odd part of $V_{v}(s, l)$ has no influence on this expected cancellation, and therefore may be assumed to vanish. In consequence, $V_{v}$ has no long-range tail, and the field $W\left(V_{v}\right)$ is infrared-regular. This brings in an important simplification: in all representations in our class there is $\pi\left(W\left(V_{v}\right)\right)=\mathrm{id}_{F} \otimes \pi_{r}\left(W\left(V_{v}\right)\right)$ and this operator is independent of $\pi(\Psi(f))=\pi_{F}(\Psi(f)) \otimes \mathrm{id}_{r}$. Our informal modified field is now $\Psi_{\pi}\left(f, V_{*}\right)=\int \overline{f(v)} \gamma \cdot v \pi_{F}(\Psi(v)) \otimes \pi_{r}\left(W\left(V_{v}\right)\right) \mathrm{d} \mu(v)$.

The use of representations for further construction is unavoidable. We shall need some general additional assumptions on their properties needed in the construction, as well as some conditions on the 'clouds' profiles $V_{*}$. We formulate these assumptions in the present section successively, and test them in a large class of representations in the next subsection.

Assumption 1. The profiles $V_{v}(s, l) \in \mathcal{V}$ are smooth functions of all their arguments $(v, s, l)$, even in $s$. For each pair of vectors $\varphi, \chi \in \mathcal{H}_{r}$ the function $v \mapsto$ $\left(\varphi, \pi_{r}\left(W\left(V_{v}\right)\right) \chi\right)_{r}$ is measurable.

Smoothness implies, in particular, that for each $V_{1}$ the function $\varphi_{V_{1}, V_{*}}(v)$ in (5.7) is smooth, and the operator $S_{V_{1}, V_{*}}$ in (5.6) is well defined in $\mathcal{S}\left(H_{+}\right)$.

Motivated by the above discussion we choose an orthonormal basis $\left\{e_{j}\right\}$ of the Hilbert space $\mathcal{K}$ formed of functions $e_{j} \in \mathcal{S}\left(H_{+}\right)$, and 'expand' $\pi_{F}(\Psi(v))$ in that basis. This leads us to the definition

$$
\Psi_{\pi}\left(f, V_{*}\right)=\sum_{j=1}^{\infty} \pi_{F}\left(\Psi\left(e_{j}\right)\right) \otimes W_{\pi_{r}}\left(V_{*}, \bar{f} \Gamma e_{j}\right),
$$

where $\Gamma$ is the operator defined by $(\Gamma f)(v)=\gamma \cdot v f(v)$, and $W_{\pi_{r}}\left(V_{*}, \rho\right)$ is defined by

$$
W_{\pi_{r}}\left(V_{*}, \rho\right)=\int \pi_{r}\left(W\left(V_{v}\right)\right) \rho(v) \mathrm{d} \mu(v),
$$

integration in the weak sense: the operators are sandwiched in $(\varphi, \cdot \chi)_{r}$ before integration. We note that $\left|\left(\varphi, \pi_{r}\left(W\left(V_{v}\right)\right) \chi\right)_{r}\right| \leq\|\varphi\|_{r}\|\chi\|_{r}$, so it is sufficient that $\rho$ be integrable. Note also that all operators $W_{\pi_{r}}\left(V_{*}, \rho\right)$ commute with each other, as all $V_{v}$ are even.

Proposition 9. The series defining $\Psi_{\pi}\left(f, V_{*}\right)$ by (5.8) converges ${ }^{*}$-strongly to a bounded operator independent of the choice of the basis $\left\{e_{j}\right\}$ in $\mathcal{S}\left(H_{+}\right)$.

Proof. If we denote by $\Psi_{\pi}^{(n)}\left(f, V_{*}\right)$ the series truncated to the first $n$ terms, set $C_{m n}=\Psi_{\pi}^{(n)}\left(f, V_{*}\right)-\Psi_{\pi}^{(m)}\left(f, V_{*}\right)$, and use the anticommutation relations for $\Psi\left(e_{j}\right)$, we find

$$
C_{m n} C_{m n}^{*}+C_{m n}^{*} C_{m n}=\operatorname{id}_{F} \otimes \sum_{j=m+1}^{n} w_{j}^{*} w_{j}
$$


where $w_{j}=W_{\pi_{r}}\left(V_{*}, \bar{f} \Gamma e_{j}\right)$. Now, using (5.9) it is easy to see that

$$
\begin{aligned}
\left(\varphi, W_{\pi_{r}}\left(V_{*}, \bar{f} \Gamma e_{j}\right) \chi\right)_{r} & =\left(f,\left(\varphi, \pi_{r}\left(W\left(V_{*}\right)\right)_{\chi}\right)_{r} e_{j}\right) \\
& =\left(\overline{\left(\varphi, \pi_{r}\left(W\left(V_{*}\right)\right) \chi\right)_{r}} f, e_{j}\right),
\end{aligned}
$$

so if we choose any orthonormal basis $\varphi_{k}$ of $\mathcal{H}_{r}$, we find

$$
\begin{aligned}
\sum_{j=1}^{\infty}\left(\chi, w_{j}^{*} w_{j} \chi\right)_{r} & =\sum_{j, k=1}^{\infty}\left|\left(\varphi_{k}, w_{j} \chi\right)_{r}\right|^{2} \\
& =\sum_{k=1}^{\infty} \int\left|\left(\varphi_{k}, \pi_{r}\left(W\left(V_{v}\right)\right) \chi\right)_{r}\right|^{2} \overline{f(v)} \gamma \cdot v f(v) \mathrm{d} \mu(v)=\|f\|^{2}\|\chi\|_{r}^{2},
\end{aligned}
$$

the last step by the Lebesgue theorem. As $\sum_{j=1}^{n} w_{j}^{*} w_{j}$ is an increasing sequence of operators, this calculation shows that $\sum_{j=1}^{\infty} w_{j}^{*} w_{j}=\|f\|^{2} \mathrm{id}_{r}$ in the $\sigma$-strong sense. This is sufficient for the ${ }^{*}$-strong convergence of the series (5.8) and the bound of the norm of the limit. The independence of the basis follows from the action of the limit operator on product vectors. It is easy to see with the use of (5.11) that

$$
\left(\xi_{1} \otimes \chi_{1}, \Psi_{\pi}\left(f, V_{*}\right) \xi_{2} \otimes \chi_{2}\right)=\left(\xi_{1}, \pi_{F}\left(\Psi\left(\overline{\left(\chi_{1}, \pi_{r}\left(W\left(V_{*}\right)\right)_{2}\right)_{r}} f\right)\right) \xi_{2}\right)_{F} .
$$

The (anti-) commutation relations of the 'dressed' Dirac fields are:

$$
\begin{aligned}
{\left[\Psi_{\pi}\left(f, V_{*}\right), \Psi_{\pi}\left(f^{\prime}, V_{*}^{\prime}\right)\right]_{+} } & =0, \\
{\left[\Psi_{\pi}\left(f, V_{*}\right), \Psi_{\pi}\left(f^{\prime}, V_{*}^{\prime}\right)^{*}\right]_{+} } & =\operatorname{id}_{F} \otimes W_{\pi_{r}}\left(V_{*}-V_{*}^{\prime}, \overline{f^{\prime}} \Gamma f\right), \\
\pi\left(W\left(V_{1}\right)\right) \Psi_{\pi}\left(f, V_{*}\right) & =\Psi_{\pi}\left(S_{V_{1}, V_{*}} f, V_{*}\right) \pi\left(W\left(V_{1}\right)\right),
\end{aligned}
$$

where $S_{V_{1}, V_{*}}$ is given, as in the heuristic introduction, by (5.7). These relations are straightforwardly calculated with the use of the definition (5.8). For the second and third identity use the technique of the above proof and the independence of basis $\left\{e_{j}\right\}$ respectively. Setting $V_{*}^{\prime}=V_{*}$ we find that dressed fields with a fixed profile $V_{*}$ satisfy the usual CAR relations among themselves. It follows thus by a standard argument (see e.g. [7]) that $\left\|\Psi_{\pi}\left(f, V_{*}\right)\right\|=\|f\|$.

To investigate the long-range behaviour of the dressed fields, we scale their radiation clouds. The profile $V_{v}$ in the element $W\left(V_{v}\right)$ may be assumed to result from a conserved current $J_{v}$ supported in $R_{\gamma, \delta}$, having vanishing asymptote, even with respect to the reflection: $J_{v}(-x)=J_{v}(x)$. As then, in loose terms, $W\left(V_{v}\right)=\exp \left[-i A\left(J_{v}\right)\right]$ and $A\left(J_{v}\right)=\int A(x) J_{v}(x) \mathrm{d}^{4} x$, scaling the electromagnetic field observable to spacelike infinity means replacing $J_{v}$ by $J_{v}^{R}(x)=R^{-3} J_{v}(x / R)$ and taking the limit $R \rightarrow \infty$ (cf. [8]). This scaling induces a simple scaling law for $V_{v}$. Thus we set

$$
V_{v}^{R}(s, l)=V_{v}(s / R, l)
$$


Assumption 2. There exist weak limits

$$
\mathrm{w}-\lim _{R \rightarrow \infty} \pi_{r}\left(W\left(V_{v}^{R}\right)\right)=\mathcal{N}_{\pi_{r}}\left(V_{v}\right) W_{\pi_{r}}^{\infty}\left(V_{v}\right),
$$

such that $W_{\pi_{r}}^{\infty}\left(V_{v}\right)$ are unitary operators in $\mathcal{H}_{r}$, and the real, positive functions $v \mapsto \mathcal{N}_{\pi_{r}}\left(V_{v}\right)>0$ are smooth and such that $1 / \mathcal{N}_{\pi_{r}}\left(V_{v}\right)$ are multipliers in $\mathcal{S}\left(H_{+}\right)$.

Note that it follows from Assumptions 1 and 2 that $\mathcal{N}_{\pi_{r}}\left(V_{v}\right) \leq 1$ and functions $v \mapsto\left(\varphi, W_{\pi_{r}}^{\infty}\left(V_{v}\right) \chi\right)$ are measurable for all $\varphi, \chi \in \mathcal{H}_{r}$. Also, the operators $W_{\pi_{r}}^{\infty}\left(V_{v}\right)$ commute with each other.

Mimicking the definitions (5.9) and (5.8) we now define

$$
\begin{aligned}
W_{\pi_{r}}^{\infty}\left(V_{*}, \rho\right) & =\int W_{\pi_{r}}^{\infty}\left(V_{v}\right) \rho(v) \mathrm{d} \mu(v), \\
\Psi_{\pi}^{\infty}\left(f, V_{*}\right) & =\sum_{j=1}^{\infty} \pi_{F}\left(\Psi\left(e_{j}\right)\right) \otimes W_{\pi_{r}}^{\infty}\left(V_{*}, \bar{f} \Gamma e_{j}\right),
\end{aligned}
$$

and note that also the analogue of (5.13) holds:

$$
\left(\xi_{1} \otimes \chi_{1}, \Psi_{\pi}^{\infty}\left(f, V_{*}\right) \xi_{2} \otimes \chi_{2}\right)=\left(\xi_{1}, \pi_{F}\left(\Psi\left(\overline{\left(\chi_{1}, W_{\pi_{r}}^{\infty}\left(V_{*}\right) \chi_{2}\right)_{r}} f\right)\right) \xi_{2}\right)_{F} .
$$

The correctness and independence of basis of the definition (5.20) is shown as in the proof of Proposition 9. It is now easy to show that (the order of limits in the second relation is irrelevant)

$$
\begin{aligned}
& \mathrm{w}-\lim _{R \rightarrow \infty} W_{\pi_{r}}\left(V_{*}^{R}, \rho / \mathcal{N}_{\pi_{r}}\left(V_{*}\right)\right)=W_{\pi_{r}}^{\infty}\left(V_{*}, \rho\right), \\
& \mathrm{w}-\lim _{R \rightarrow \infty} \lim _{R^{\prime} \rightarrow \infty} W_{\pi_{r}}\left(V_{*}^{R}-V_{*}^{\prime R^{\prime}}, \rho /\left[\mathcal{N}_{\pi_{r}}\left(V_{*}\right) \mathcal{N}_{\pi_{r}}\left(V_{*}^{\prime}\right)\right]\right) \\
& \quad=\int W_{\pi_{r}}^{\infty}\left(V_{v}\right) W_{\pi_{r}}^{\infty}\left(V_{v}^{\prime}\right)^{*} \rho(v) \mathrm{d} \mu(v), \\
& \mathrm{w}-\lim _{R \rightarrow \infty} \Psi_{\pi}\left(f / \mathcal{N}_{\pi_{r}}\left(V_{*}\right), V_{*}^{R}\right)=\Psi_{\pi}^{\infty}\left(f, V_{*}\right) ;
\end{aligned}
$$

for the last relation use (5.13) and the uniform boundedness of the norms of the operators under the limit. To find the (anti-) commutation relations of the dressed fields, we use their representation (5.24) and the relations (5.14) (5.16), with the use of $(5.23)$ on the r.h.s. of (5.15). Setting now $V_{*}^{\prime}=V_{*}$ we find

$$
\begin{aligned}
{\left[\Psi_{\pi}^{\infty}\left(f, V_{*}\right), \Psi_{\pi}^{\infty}\left(f^{\prime}, V_{*}\right)\right]_{+} } & =0 \\
{\left[\Psi_{\pi}^{\infty}\left(f, V_{*}\right), \Psi_{\pi}^{\infty}\left(f^{\prime}, V_{*}\right)^{*}\right]_{+} } & =\left(f, f^{\prime}\right)_{\mathcal{K}} \mathrm{id}, \\
\pi\left(W\left(V_{1}\right)\right) \Psi_{\pi}^{\infty}\left(f, V_{*}\right) & =\Psi_{\pi}^{\infty}\left(S_{V_{1}, V_{*}}^{\infty} f, V_{*}\right) \pi\left(W\left(V_{1}\right)\right),
\end{aligned}
$$

where

$$
\begin{aligned}
\left(S_{V_{1}, V_{*}}^{\infty} f\right)(v) & =\exp \left[i \varphi_{V_{1}, V_{*}}^{\infty}(v)\right] f(v), \\
\varphi_{V_{1}, V_{*}}^{\infty}(v) & =-\frac{e}{4 \pi} \int \frac{v \cdot \Delta V_{1}(l)}{v \cdot l} \mathrm{~d}^{2} l+\frac{1}{2 \pi} \int V_{v}(0, l) \cdot \Delta V_{1}(l) \mathrm{d}^{2} l .
\end{aligned}
$$


To show (5.27), one notes first that $\lim _{R \rightarrow \infty} \varphi_{V_{1}, V_{*}^{R}}(v)=\varphi_{V_{1}, V_{*}}^{\infty}(v)$ and then observes that while taking the weak limit of $\Psi_{\pi}\left(S_{V_{1}, V_{*}^{R}} f / \mathcal{N}_{\pi_{r}}\left(V_{*}\right), V_{*}^{R}\right)$ one can replace $S_{V_{1}, V_{*}^{R}}$ by $S_{V_{1}, V_{*}}^{\infty}$ as the difference vanishes in norm.

We note that the dependence of $\varphi_{V_{1}, V_{*}}^{\infty}(v)$ on $V_{1}$ is only through its infrared tail $\Delta V_{1}$. In spacetime terms it means that the dependence on the test current $J_{1}$ giving rise to $V_{1}$ is only through its asymptote $J_{1}^{\text {as }}$, which may be assumed to be of the form $J_{1}^{\text {as }}(z)=z \rho_{1}(z)$, in accordance with Proposition 4 . Thus using (4.14) we can write

$$
\begin{aligned}
\varphi_{V_{1}, V_{*}}^{\infty}(v) & =\int \rho_{1}(z) F_{v}(z) \mathrm{d} \nu(z), \\
F_{v}(z) & =\frac{1}{2 \pi} \int \frac{1}{z \cdot l} z \cdot\left[V_{v}(0, l)-\frac{e v}{2 v \cdot l}\right] \mathrm{d}^{2} l,
\end{aligned}
$$

the second integral in the principal value sense.

The negative result mentioned at the beginning of Sect. 5 is now the following.

Theorem 10. There is no choice of profiles $V_{v}(0, l)$ such that $S_{V_{1}, V_{*}}^{\infty} f=f$ would hold for any test function $f$ and for all $J_{1}^{\text {as }}(z)=z \rho_{1}(z)$ supported in any given fixed symmetrical spacelike cone.

Proof. The asymptote $\rho_{1}(z)$ is subject to two conditions: it must be an even function and satisfy $\int \rho_{1}(z) \mathrm{d} \nu(z)=0$ (cf. (4.5)). The only way to achieve $\exp \left[i \varphi_{V_{1}, V_{*}}^{\infty}(v)\right]=1$ for some $v$ and all admissible $\rho_{1}$ supported in a given symmetrical spacelike cone would be that $F_{v}(z)=$ const. on the patch of hyperboloid defining this cone (note that $F_{v}(z)$ is also even). This, however, is impossible for the following reason. It is easily seen that $F_{v}(z)$ extends naturally to an even, homogeneous function $F_{v}(x)$ of degree 0 for all $x^{2}<0$ (by simply replacing $z$ by $x$ in $(5.31)$ ). Now $F_{v}(z)=$ const. in a patch iff $F_{v}(x)=$ const. in the corresponding cone. This, however, is impossible, as we shall see that $\square F_{v}(x)=2 e / x^{2}$. To show this, we first use the result of Appendix A of [3]. Each possible profile $V_{v}(0, l)$ must be orthogonal to $l$ and thus satisfies the conditions on $V(l)$ of this Appendix. Using Eq. (A.4) one finds

$$
\begin{aligned}
F_{v}^{(1)}(x) & \equiv \frac{1}{2 \pi} \int \frac{x \cdot V_{v}(0, l)}{x \cdot l} \mathrm{~d}^{2} l \\
& =-\frac{1}{2 \pi} \int \partial \cdot V_{v}(0, l) \log \frac{|x \cdot l|}{v \cdot l} \mathrm{~d}^{2} l+\frac{1}{2 \pi} \int \frac{v \cdot V_{v}(0, l)}{v \cdot l} \mathrm{~d}^{2} l .
\end{aligned}
$$

This implies $\square F_{v}^{(1)}(x)=0$. On the other hand one explicitly calculates

$$
\begin{aligned}
F_{v}^{(2)}(x) & =-\frac{e v \cdot x}{4 \pi} \int \frac{\mathrm{d}^{2} l}{x \cdot l v \cdot l} \\
& =-e \frac{v \cdot x}{\sqrt{(v \cdot x)^{2}-x^{2}}} \operatorname{artanh} \frac{v \cdot x}{\sqrt{(v \cdot x)^{2}-x^{2}}}
\end{aligned}
$$

and $\square F_{v}^{(2)}(x)=2 e / x^{2}$. 
This result shows that it is impossible to choose $V_{v}(s, l)$ in such a way that the exponential factor in (5.28) vanishes for all test functions and test currents supported in symmetrical spacelike cones. However, one can find $V_{v}(s, l)$ which makes this exponential factor independent of $v$. Let

$$
V_{v}(s, l)=\left(\frac{e}{2}\right)\left(\frac{v}{v \cdot l}-\frac{t}{t \cdot l}\right) \eta\left(\frac{s}{t \cdot l}\right),
$$

where $t$ is a timelike unit vector and $\eta(s)$ is a smooth function satisfying: $0 \leq \eta(s) \leq 1, \eta(0)=1, \eta(s)=\eta(-s)$ and there exist $s_{0}>0$ such that $\eta(s)=0$ for $s>s_{0}$. For this profile, if it satisfies Assumptions 1 and 2 (beside smoothness, which is obvious), it follows that:

$$
\varphi_{V_{1}, V_{*}}^{\infty}=-\frac{e}{4 \pi} \int \frac{t \cdot \Delta V_{1}(l)}{t \cdot l} \mathrm{~d}^{2} l, \quad S_{V_{1}, V_{*}}^{\infty}=\exp \left[i \varphi_{V_{1}, V_{*}}^{\infty}\right] \mathrm{id} .
$$

The commutation relation (5.27) and its adjoint take now the following simple form:

$$
\begin{aligned}
\pi\left(W\left(V_{1}\right)\right) \Psi_{\pi}^{\infty}\left(f, V_{*}\right) & =e^{i \varphi_{V_{1}, V_{*}}^{\infty} \Psi_{\pi}^{\infty}\left(f, V_{*}\right) \pi\left(W\left(V_{1}\right)\right),} \\
\pi\left(W\left(V_{1}\right)\right) \Psi_{\pi}^{\infty}\left(f, V_{*}\right)^{*} & =e^{-i \varphi_{V_{1}, V_{*}}^{\infty} \Psi_{\pi}^{\infty}\left(f, V_{*}\right)^{*} \pi\left(W\left(V_{1}\right)\right) .}
\end{aligned}
$$

It is now possible to restrict the scope of test functions $f$ to those resulting from compactly supported four-spinor test fields $\chi$ in (5.3). Then the observables $\Psi_{\pi}^{\infty}\left(f, V_{*}\right)^{*} \Psi_{\pi}^{\infty}\left(f^{\prime}, V_{*}\right)$ form a local net commuting with the electromagnetic field, with localization determined by the union of the supports of $\chi$ and $\chi^{\prime}$. These are the asymptotic incarnations, in our model, of the quantities discussed at the beginning of Sect. 5 .

Assumption 3. For any two profiles $V_{v}, V_{v}^{\prime}$ of the form (5.34) (with possibly different vectors $t$ and functions $\eta)$ the unitary operator $W_{\pi_{r}}^{\infty}\left(V_{v}\right) W_{\pi_{r}}^{\infty}\left(V_{v}^{\prime}\right)^{*}$ formed by the operators defined by Assumption 2 is independent of $v$.

With this assumption it is now easy to see that the observables defined above do not depend on a particular choice of the profile $V_{v}$ in the class (5.34).

\subsection{Special Choice of Representation}

In this subsection we show that Assumptions 1, 2 and 3 are fulfilled for profiles (5.34) in a class of representations $\pi_{r}$ in (3.26) constructed in earlier papers.

Consider the vector space of equivalence classes of real, smooth vector functions $f_{a}(l)$ on the cone, homogeneous of degree -1 , with $l \cdot f(l)=0$. The equivalence relation is introduced by: $f_{1} \sim f_{2} \Leftrightarrow f_{1 a}(l)=f_{2 a}(l)+\beta(l) l_{a}$. The completion of this space with respect to the scalar product

$$
\left(f_{1}, f_{2}\right)_{0}=-\int f_{1}(l) \cdot f_{2}(l) \mathrm{d}^{2} l
$$

is a real Hilbert space denoted $\mathcal{H}_{0}$. The closure of the subspace of (equivalence classes of) smooth functions satisfying $L \wedge f=0$ forms a Hilbert space denoted by $\mathcal{H}_{I R}$. Let $H(s, l)$ be a homogeneous of degree 0 , smooth function, such that $\lim _{s \rightarrow \pm \infty} H(s, l)= \pm 1$ and $\dot{H}(s, l)$ satisfies the falloff condition analogous to 
(3.3). We denote $h(s, l)=\pi \dot{H}(s, l)$ and fix notation for Fourier transform with respect to $s$ by

$$
\widetilde{h}(\omega, l)=\frac{1}{2 \pi} \int e^{i \omega s} h(s, l) \mathrm{d} s,
$$

so $\widetilde{h}(0, l)=1$. Following the notation of [1] and [3] we set

$$
p(\dot{V})=\tilde{\dot{V}}(0, l)=\frac{1}{2 \pi} \Delta V,
$$

the long range characteristic of $V(s, l)$, and denote by $r_{h}(\dot{V})$ the orthogonal projection of $\frac{1}{2} \int \dot{V} H(s, l) \mathrm{d} s$ onto $\mathcal{H}_{I R}$. We split function $V(s, l)$ into the IR-regular and IR-singular part by setting:

$$
\begin{aligned}
\widetilde{\dot{V}}(\omega, l) & =[\widetilde{\dot{V}}(\omega, l)-\widetilde{\dot{V}}(0, l) \widetilde{h}(\omega, l)]+\widetilde{\dot{V}}(0, l) \widetilde{h}(\omega, l) \\
& =\widetilde{\dot{V}}_{\mathrm{reg}}(\omega, l)+\widetilde{\dot{V}}(0, l) \widetilde{h}(\omega, l) .
\end{aligned}
$$

In particular, $p(\dot{V})=0$ means that $\dot{V}$ is IR-regular, i.e. the field has no 'long range tail'.

Further, we consider the Weyl algebra generated by the elements $w(g \oplus k)$, where $g \oplus k$ belongs to the vector space $\mathcal{C}_{I R}^{\infty} \oplus \mathcal{C}_{I R}^{\infty}\left(\mathcal{C}_{I R}^{\infty}:=\mathcal{C}^{\infty} \cap \mathcal{H}_{I R}\right.$, differentiability is understood in the sense of $L_{a b}$ ) with the symplectic structure:

$$
\left\{g_{1} \oplus k_{1}, g_{2} \oplus k_{2}\right\}_{I R}:=\left(g_{1}, k_{2}\right)_{I R}-\left(k_{1}, g_{2}\right)_{I R} .
$$

Algebraic relations satisfied by elements $w(g \oplus k)$ are

$$
\begin{aligned}
w\left(g_{1} \oplus k_{1}\right) w\left(g_{2} \oplus k_{2}\right) & =e^{-\frac{i}{2}\left\{g_{1} \oplus k_{1}, g_{2} \oplus k_{2}\right\}_{I R}} w\left(\left(g_{1}+g_{2}\right) \oplus\left(k_{1}+k_{2}\right)\right) \\
w(g \oplus k)^{*} & =w(-(g \oplus k)) .
\end{aligned}
$$

Let $\pi_{\text {sing }}$ be a cyclic representation of this algebra derived by GNS construction from the state

$$
\omega_{\text {sing }}(\omega(g \oplus k))=\exp \left(-\frac{1}{4}\left(g, C^{-1} g\right)_{I R}-\frac{1}{4}(k, C k)_{I R}\right),
$$

with the corresponding Hilbert space $\mathcal{H}_{\text {sing }}$ and the cyclic vector $\Omega_{\text {sing. }}$. Here $C$ is any positive, trace-class operator such that $\mathcal{C}_{I R}^{\infty} \subset C^{1 / 2} \mathcal{H}_{I R},{\overline{C^{-1 / 2} \mathcal{C}_{I R}^{\infty}}}^{\mathcal{H}_{I R}}=$ $\mathcal{H}_{I R}$. Denote by $\pi_{0}$ the standard positive energy Fock representation of infrared-regular fields, generated by GNS construction from the vacuum state

$$
\begin{aligned}
\omega_{0}\left(W\left(V_{\text {reg }}\right)\right) & =\exp \left(-\frac{1}{2} F\left(\dot{V}_{\text {reg }}, \dot{V}_{\text {reg }}\right)\right), \\
F\left(\dot{V}_{1}, \dot{V}_{2}\right) & =\int_{\omega \geq 0}\left(-\overline{\widetilde{V}_{1}(\omega, l)} \cdot \tilde{\dot{V}}_{2}(\omega, l)\right) \frac{\mathrm{d} \omega}{\omega} \mathrm{d}^{2} l,
\end{aligned}
$$

with the corresponding Hilbert space $\mathcal{H}_{\text {reg }}$ and cyclic vector $\Omega_{0}$. Then the formula

$$
\pi_{r}(W(V))=\pi_{\text {sing }}\left(w\left(p(\dot{V}) \oplus r_{h}(\dot{V})\right)\right) \otimes \pi_{0}\left(W\left(V_{\text {reg }}\right)\right)
$$


determines a regular, translationally covariant positive energy representation of $\mathcal{B}^{-}$on $\mathcal{H}_{r}=\mathcal{H}_{\text {sing }} \otimes \mathcal{H}_{\text {reg }}$ [1]. Now one has to prove that Assumptions 1,2 and 3 are fulfilled for this choice of $\pi_{r}$.

It was shown in [1] that the representation $\pi_{r}$ does not depend on the concrete shape of $H(s, l)$. Therefore, for the convenience of the proof of proposition 11, we shall assume, from now on, a special choice of this function. We put $H(s, l)=H_{t}(s / t \cdot l)$ for a timelike unit vector $t$, and a smooth function $H_{t}$ such that for some $u_{0}>0$ there is $H_{t}(u)=1$ for $u>u_{0}$, and $H_{t}(u)=-1$ for $u<-u_{0}$.

Proposition 11. For the representation $\pi_{r}$ defined by (5.46) and the profiles $V_{v}$ given by (5.34), Assumptions 1, 2 and 3 are satisfied.

Proof. (Assumption 1) To prove the measurability, it suffices to show that $\left(y, \pi_{r}\left(W\left(V_{v}\right)\right) x\right)_{r}$ is continuous in $v$ for vectors from a total set, those of the form

$$
\begin{aligned}
& x=\pi_{\text {sing }}\left(w\left(g_{1} \oplus k_{1}\right)\right) \Omega_{\text {sing }} \otimes \pi_{0}\left(W\left(V_{1}\right)\right) \Omega_{0}, \\
& y=\pi_{\text {sing }}\left(w\left(g_{2} \oplus k_{2}\right)\right) \Omega_{\text {sing }} \otimes \pi_{0}\left(W\left(V_{2}\right)\right) \Omega_{0},
\end{aligned}
$$

where $V_{i}, i=1,2$, are IR-regular. As $V_{v}$ is IR-regular, so

$$
\pi_{r}\left(W\left(V_{v}\right)\right)=\pi_{\text {sing }}\left(w\left(0 \oplus r_{h}\left(\dot{V}_{v}\right)\right)\right) \otimes \pi_{0}\left(W\left(V_{v}\right)\right) .
$$

One obtains:

$$
\begin{aligned}
\left(y, \pi_{r}\left(W\left(V_{v}\right)\right) x\right)_{r}= & \omega_{\text {sing }}\left(w\left(g_{2} \oplus k_{2}\right)^{*} w\left(0 \oplus r_{h}\left(\dot{V}_{v}\right)\right) w\left(g_{1} \oplus k_{1}\right)\right) \\
& \times \omega_{0}\left(W\left(-V_{2}\right) W\left(V_{v}\right) W\left(V_{1}\right)\right) .
\end{aligned}
$$

From the algebraic relations it follows that:

$$
\begin{aligned}
\omega_{0}\left(W\left(-V_{2}\right) W\left(V_{v}\right) W\left(V_{1}\right)\right)= & \exp \left[-\frac{1}{2} F\left(\dot{V}_{1}-\dot{V}_{2}+\dot{V}_{v}, \dot{V}_{1}-\dot{V}_{2}+\dot{V}_{v}\right)\right. \\
& \left.-\frac{i}{2}\left\{V_{v}, V_{1}+V_{2}\right\}-\frac{i}{2}\left\{V_{1}, V_{2}\right\}\right] .
\end{aligned}
$$

Because $F\left(\dot{V}_{v}, \dot{V}_{v}\right)$ and $F\left(\dot{V}_{v}, \dot{V}_{k}\right)(k=1,2)$, as easily shown, are smooth in $v$, so is the r.h.s. of (5.49). Now we turn to $\omega_{\text {sing. Using }}(5.41),(5.42)$ and (5.43), one finds:

$$
\begin{aligned}
& \omega_{\text {sing }}\left(w\left(g_{2} \oplus k_{2}\right)^{*} w\left(0 \oplus r_{h}\left(\dot{V}_{v}\right)\right) w\left(g_{1} \oplus k_{1}\right)\right) \\
& =\exp \left[-\frac{1}{4}\left(\Delta g, C^{-1} \Delta g\right)_{I R}-\frac{1}{4}\left(\Delta k+r_{h}\left(\dot{V}_{v}\right), C\left[\Delta k+r_{h}\left(\dot{V}_{v}\right)\right]\right)_{I R}\right] \\
& \quad \times \exp \left[\frac{i}{2}\left(r_{h}\left(\dot{V}_{v}\right), g_{1}+g_{2}\right)_{I R}+\frac{i}{2}\left(g_{2}, k_{1}\right)_{I R}-\frac{i}{2}\left(g_{1}, k_{2}\right)_{I R}\right],
\end{aligned}
$$

where $\Delta g=g_{1}-g_{2}, \Delta k=k_{1}-k_{2}$. To prove that the r.h.s of (5.50) is indeed a continuous function in $v$, it suffices to show that terms of the form: $\left(r_{h}\left(\dot{V}_{v}\right), C r_{h}\left(\dot{V}_{v}\right)\right)_{I R},\left(r_{h}\left(\dot{V}_{v}\right), k\right)_{I R},\left(k, C r_{h}\left(\dot{V}_{v}\right)\right)_{I R}$ are continuous in $v$ for 
$k \in \mathcal{C}_{I R}^{\infty}$. As $C$ is a bounded operator, it is sufficient to show that $r_{h}\left(\dot{V}_{v}\right)$, as an element of $\mathcal{H}_{I R}$, is norm-continuous in $v$. Since

$$
\frac{1}{2} \int \dot{V}_{v}(s, l) H_{t}\left(\frac{s}{t \cdot l}\right) \mathrm{d} s=\frac{e}{4} \int \dot{\eta}(u) H_{t}(u) \mathrm{d} u\left(\frac{v}{v \cdot l}-\frac{t}{t \cdot l}\right)=r_{h}\left(\dot{V}_{v}\right)(l),
$$

we have

$$
\left\|r_{h}\left(\dot{V}_{v}\right)-r_{h}\left(\dot{V}_{v^{\prime}}\right)\right\|_{I R}^{2}=\left(\frac{e}{4} \int \dot{\eta}(u) H_{t}(u) \mathrm{d} u\right)^{2}\left[-\int\left(\frac{v}{v \cdot l}-\frac{v^{\prime}}{v^{\prime} \cdot l}\right)^{2} \mathrm{~d}^{2} l\right] .
$$

The last integral can be calculated explicitly:

$$
\begin{aligned}
& -\int\left(\frac{v}{v \cdot l}-\frac{v^{\prime}}{v^{\prime} \cdot l}\right)^{2} \mathrm{~d}^{2} l=\int\left[2 \frac{v \cdot v^{\prime}}{(v \cdot l)\left(v^{\prime} \cdot l\right)}-\frac{1}{(v \cdot l)^{2}}-\frac{1}{\left(v^{\prime} \cdot l\right)^{2}}\right] \mathrm{d}^{2} l \\
& =8 \pi\left\{\frac{v \cdot v^{\prime}}{\sqrt{\left(v \cdot v^{\prime}\right)^{2}-1}} \log \left(v \cdot v^{\prime}+\sqrt{\left(v \cdot v^{\prime}\right)^{2}-1}\right)-1\right\} .
\end{aligned}
$$

Because (5.53) converges to 0 for $v \rightarrow v^{\prime}, r_{h}\left(\dot{V}_{v}\right)$ is norm continuous. Finally we can conclude that (5.50) is a continuous function of $v$. This ends the proof of Assumption 1.

(Assumptions 2 and 3) First we show the existence of the weak limit $\mathrm{w}-\lim _{R \rightarrow \infty} \pi_{r}\left(W\left(V_{v}^{R}\right)\right)$. The norms of $\pi_{r}\left(W\left(V_{v}^{R}\right)\right)$ are uniformly bounded, so it is sufficient to obtain the weak limit for operators sandwiched between vectors from a total set chosen as in the proof of Assumption 1. We have to investigate the limit of the expressions (5.49) and (5.50) in which $V_{v}$ has been replaced by $V_{v}^{R}$, for $R \rightarrow \infty$. From (5.17) and (5.38) one has $\widetilde{\dot{V}}_{v}^{R}(\omega, l)=\widetilde{\dot{V}}_{v}(R \omega, l)$. As $\widetilde{\dot{V}}_{k}(0, l)=0, k=1,2$, it follows by the Lebesgue dominated convergence theorem that $\lim _{R \rightarrow \infty} F\left(\dot{V}_{v}^{R}, \dot{V}_{k}\right)=0$ (see (5.45)), and since $\left\{V_{v}^{R}, V_{k}\right\}=2 \mathfrak{I m}\left(F\left(\dot{V}_{v}^{R}, \dot{V}_{k}\right)\right)$, also $\lim _{R \rightarrow \infty}\left\{V_{v}^{R}, V_{k}\right\}=0$. On the other hand, by a change of the integration variable $\omega$ one finds

$$
F\left(\dot{V}_{v}^{R}, \dot{V}_{v}^{R}\right)=F\left(\dot{V}_{v}, \dot{V}_{v}\right) .
$$

In this way, for the scaled version of (5.49), we obtain:

$$
\lim _{R \rightarrow \infty} \omega_{0}\left(W\left(-V_{2}\right) W\left(V_{p}^{R}\right) W\left(V_{1}\right)\right)=\mathcal{N}_{\pi_{r}}\left(V_{v}\right) \omega_{0}\left(W\left(-V_{2}\right) W\left(V_{1}\right)\right),
$$

where

$$
\mathcal{N}_{\pi_{r}}\left(V_{v}\right)=\exp \left(\frac{1}{2} \int_{\omega \geq 0} \overline{\tilde{\dot{V}}_{v}(\omega, l)} \cdot \widetilde{\dot{V}}_{v}(\omega, l) \frac{\mathrm{d} \omega}{\omega} \mathrm{d}^{2} l\right)
$$

Thus

$$
\mathrm{W}-\lim _{R \rightarrow \infty} \pi_{0}\left(W\left(V_{v}^{R}\right)\right)=\mathcal{N}_{\pi_{r}}\left(V_{v}\right) \text { id }
$$


For the IR-singular part we note that

$$
\lim _{R \rightarrow \infty}\left\|r_{h}\left(\dot{V}_{v}^{R}\right)+V_{v}(0, .)\right\|_{I R}=0,
$$

which is easily shown with the use of (5.51). Thus using the scaled version of (5.50) we find

$$
\mathrm{w}-\lim _{R \rightarrow \infty} \pi_{\text {sing }}\left(w\left(0 \oplus r_{h}\left(\dot{V}_{v}^{R}\right)\right)\right)=\pi_{\text {sing }}\left(w\left(0 \oplus-V_{v}(0, .)\right)\right) .
$$

Therefore, we can finally conclude that the relation (5.18) is satisfied, with $\mathcal{N}_{\pi_{r}}$ given by (5.56), and

$$
W_{\pi_{r}}^{\infty}\left(V_{v}\right)=\pi_{\text {sing }}\left(w\left(0 \oplus-V_{v}(0, .)\right) \otimes \mathrm{id} .\right.
$$

This form of these operators ensures that Assumption 3 is satisfied. After a suitable change of variables one finds that the factor function has the form

$$
\mathcal{N}_{\pi_{r}}\left(V_{v}\right)=\exp \left(\frac{e^{2}}{8} \int_{u \geq 0} u|\widetilde{\eta}(u)|^{2} \mathrm{~d} u \int\left(\frac{v}{v \cdot l}-\frac{t}{t \cdot l}\right)^{2} \mathrm{~d}^{2} l\right),
$$

where $\widetilde{\eta}$ is the Fourier transform of $\eta$ defined as in (5.38). Using (5.53) we obtain:

$\mathcal{N}_{\pi_{r}}\left(V_{v}\right)=\exp \left\{-c\left[\frac{v \cdot t}{\sqrt{(v \cdot t)^{2}-1}} \log \left(v \cdot t+\sqrt{(v \cdot t)^{2}-1}\right)-1\right]\right\}$,

where $c>0$ is a constant. The function $v \mapsto \mathcal{N}_{\pi_{r}}\left(V_{v}\right)$ is smooth and for $v^{0} \rightarrow \infty$ we have: $1 / \mathcal{N}_{\pi_{r}}\left(V_{v}\right) \sim \operatorname{const}\left(v^{0}\right)^{c}$, with similar estimates for derivatives. This proves that $1 / \mathcal{N}_{\pi_{r}}\left(V_{v}\right)$ are multipliers in $\mathcal{S}\left(H_{+}\right)$.

\section{Conclusions}

The algebra proposed earlier for the description of asymptotic fields in spinor electrodynamics incorporates Gauss' law and thus has good chances to form (at least a substantial part of) a consistent model of the long-range behavior of QED. We have found here how to give the elements of this field algebra localization in regions contained in an arbitrarily chosen time slice 'fattening towards edges'. Compact localization regions may be chosen only for infraredregular electromagnetic fields. Both infrared-singular electromagnetic fields as well as charged fields have always localization regions extending to spacelike infinity. However, the infrared singular electromagnetic fields may be decomposed into fields localized in arbitrarily 'thin' fattened symmetrical spacelike cones. On the other hand we have found that there is no way of attaching an infrared cloud to the charged field so as to localize it in such region, at least in a wide class of representations which satisfy some natural general conditions. Nevertheless, we have also shown that compactly supported observables may be formed by simple multiplication of appropriately dressed charged fields with compensating charges.

The lack of spacelike-cone localization of dressed Dirac fields in the present model seems to be nonstandard, as already mentioned in Introduction 
and Sect. 5. One could object that the model, although it incorporates global Gauss' law, still lacks some additional asymptotic electromagnetic variables. The construction of the model suggests that in such case the variables would have to originate as limits of gauge-dependent local electromagnetic potentials. However, whether the model is indeed incomplete can only be decided by finding its place in a formulation of fully interacting electrodynamics. In particular, it would be interesting to formulate a perturbative electrodynamics incorporating some nonperturbative infrared aspects of the present model.

On the other hand, we would like to stress once more a physically important aspect of the model considered here. Our fundamental fermion fields are genuinely charged, satisfying Gauss' law even before 'dressing'. Dressing is considered for the sake of inducing a certain localization of these fields, as well as an auxiliary step in the construction of bi-fermion observables. Simplified as the model is, it is at the same time non-perturbative.

This is to be contrasted with all forms of 'dressing' of fermion fields in local formulations of QED. There, in the indefinite metric space (GuptaBleuler), local Dirac fields cannot carry physical charge, as they commute with the electric flux at spatial infinity. After constructing a perturbative solution of an initial theory formulated in such space, one attempts then, by the addition of Lorenz condition and nonlocal dressing of charged fields, to restore Maxwell equations and transport the theory into a Hilbert space of physical vector states. The dressing takes the form of a formal local gauge transformation in which the gauge function is constructed with the use of electromagnetic potential (see e.g. [9]). In an Ansatz put forward by Dirac this has the following form:

$$
\Psi(x)=\exp [i e G(x)] \psi(x), \quad \mathcal{A}(x)=A(x)-\partial G(x)
$$

where $G(x)=\int r^{a}(x-y) A_{a}(y) \mathrm{d}^{4} y$; here $r^{a}(x)$ is a vector distribution satisfying $\partial_{a} r^{a}(x)=\delta^{4}(x)$. Within perturbative approach to QED this idea has been implemented most rigorously in the 'axiomatic perturbative' formulation by Steinmann [6]. In this approach the above tentative transformation is carried out not on the level of fields, but rather Wightman functions. As argued by Steinmann, the results are insensitive to a choice of a particular form of the distribution $r^{a}$. And as among such distributions are some with supports in spacelike cones, one can argue that in this way charged fields may be pushed into such regions.

These constructions, rigorous as they are within the limits of the procedure followed in this approach, are not without weak points. First, not only the local interaction, but also the dressing exponent is treated perturbatively; this is admitted by Steinmann himself to be an obstacle to a completely reliable representation of the infrared problems. Secondly, the dressing transformation (6.1) is infrared-singular and cannot be performed in this form even at the level of Wightmann functions; the actual way it is done, is via an effective spatial truncation followed by an adiabatic limit. However, precisely these two points are of critical importance for the infrared problem. 
Finally, we want to comment on our choice of representations. One cannot exclude that the use of some more infrared singular representations would modify our results. That localization may be improved 'in front of' infravacua (KPR-type representations [10]) has been shown by Kuhnhardt [11] in a scalar model due to Buchholz et al. [12]. One of the main motivations for the introduction of such more singular representations of free electromagnetic fields is the fact that they may be stable under the addition of radiation fields produced in scattering processes. However, in this connection we want to mention two facts on the asymptotic model considered here. First, it has been shown in [3] that representations discussed above in Sect. 5.3 do suffice to absorb radiation fields produced by a classical current. Second, in this model the asymptotic fields are not completely decoupled, and the electric flux at spatial infinity is due both to free as well as Coulomb parts. However, the electric flux of the total field at infinity is an invariant characteristic of the process, not changing with time (the asymptotic flux depends on the spacelike direction, but, in fact, is invariant under any finite spacetime translation of the point from which we go to spacelike infinity). This is a fact in classical theory, and should be also expected in the full quantum theory.

Open Access. This article is distributed under the terms of the Creative Commons Attribution Noncommercial License which permits any noncommercial use, distribution, and reproduction in any medium, provided the original author(s) and source are credited.

\section{Appendix}

We prove here the relation (4.25). Let $y \in R_{\delta}$ and $|x-y| \leq \gamma$ and denote $\kappa=\sqrt{\left(1+\delta^{2}\right) /\left(1-\delta^{2}\right)},-y^{2}=r^{2}, r>0$. Then

$$
\left|x^{2}-y^{2}\right| \leq\left|(x-y)^{2}\right|+2|y \cdot(x-y)| \leq \gamma^{2}+2 \gamma \kappa r
$$

where we have used (2.12). Thus $-r^{2}-2 \gamma \kappa r-\gamma^{2} \leq x^{2} \leq-r^{2}+2 \gamma \kappa r+\gamma^{2}$. Consider now two cases.

(i) $y^{2}+R^{2} \geq 0$ and $x^{2}+R^{2} \leq 0$.

It follows that $R^{2}-r^{2} \geq 0$ and $-r^{2}-2 \gamma \kappa r-\gamma^{2}+R^{2} \leq 0$, so $r \in\left\langle R-R_{1}, R\right\rangle$, with $R_{1}=\gamma \kappa$ (although not the whole interval is covered).

(ii) $y^{2}+R^{2} \leq 0$ and $x^{2}+R^{2} \geq 0$.

It follows that $R^{2}-r^{2} \leq 0$ and $-r^{2}+2 \gamma \kappa r+\gamma^{2}+R^{2} \geq 0$, so $r \in\left\langle R, R+R_{2}\right\rangle$, with $R_{2}=\gamma\left(\kappa+\sqrt{\kappa^{2}+1}\right.$ ) (with the same remark as above).

Summarizing, we have that from $\left(x^{2}+R^{2}\right)\left(y^{2}+R^{2}\right) \leq 0$ it follows $-\left(R+R_{2}\right)^{2} \leq y^{2} \leq-\left(R-R_{1}\right)^{2}$ for $R \geq R_{1}$, which implies (4.25). 


\section{References}

[1] Herdegen, A.: Semidirect product of CCR and CAR algebras and asymptotic states in quantum electrodynamics. J. Math. Phys. 39, 1788 (1998)

[2] Herdegen, A.: Asymptotic algebra of quantum electrodynamics. Acta Phys. Pol. B 36, 35 (2005)

[3] Herdegen, A.: Infrared problem and spatially local observables in electrodynamics. Ann. H. Poincaré 9, 373 (2008)

[4] Herdegen, A.: Long-range effects in asymptotic fields and angular momentum of classical field electrodynamics. J. Math. Phys. 36, 4044 (1995)

[5] Buchholz, D.: The physical state space of quantum electrodynamics. Commun. Math. Phys. 85, 49 (1982)

[6] Steinmann, O.: Perturbative Quantum Electrodynamics and Axiomatic Field Theory. Springer, Berlin (2000)

[7] Bratteli, O., Robinson, D.W.: Operator Algebras and Quantum Statistical Mechanics, vol. II. Springer, Berlin (1996)

[8] Buchholz, D.: Gauss' law and the infraparticle problem. Phys. Lett. B174, 331 (1986)

[9] Symanzik, K.: Lectures on Lagrangian Quantum Field Theory. Lecture Notes from University of Islamabad, Islamabad (1968)

[10] Kraus, K., Polley, L., Reents, G.: Models for infrared dynamics: I Classical currents. Ann. Inst. H. Poincaré A26, 109 (1977)

[11] Kunhardt, W.: On infravacua and the localization of sectors. J. Math. Phys. 39, 6353 (1998)

[12] Buchholz, D., Doplicher, S., Morchio, G., Roberts, J.E., Strocchi, F.: A model for charges of electromagnetic type. In: Doplicher, S. et al. (eds.) Operator Algebras and Quantum Field Theory, pp. 647. International Press, Cambridge (1997)

Andrzej Herdegen

Institute of Physics

Jagiellonian University

Reymonta 4

30-059 Cracow, Poland

e-mail: herdegen@th.if.uj.edu.pl

Katarzyna Rejzner

Institute for Theoretical Physics

Hamburg University

Luruper Chaussee 149

22761 Hamburg, Germany

e-mail: katarzyna.rejzner@desy.de

Communicated by Klaus Fredenhagen.

Received: May 28, 2010.

Accepted: February 11, 2011. 